
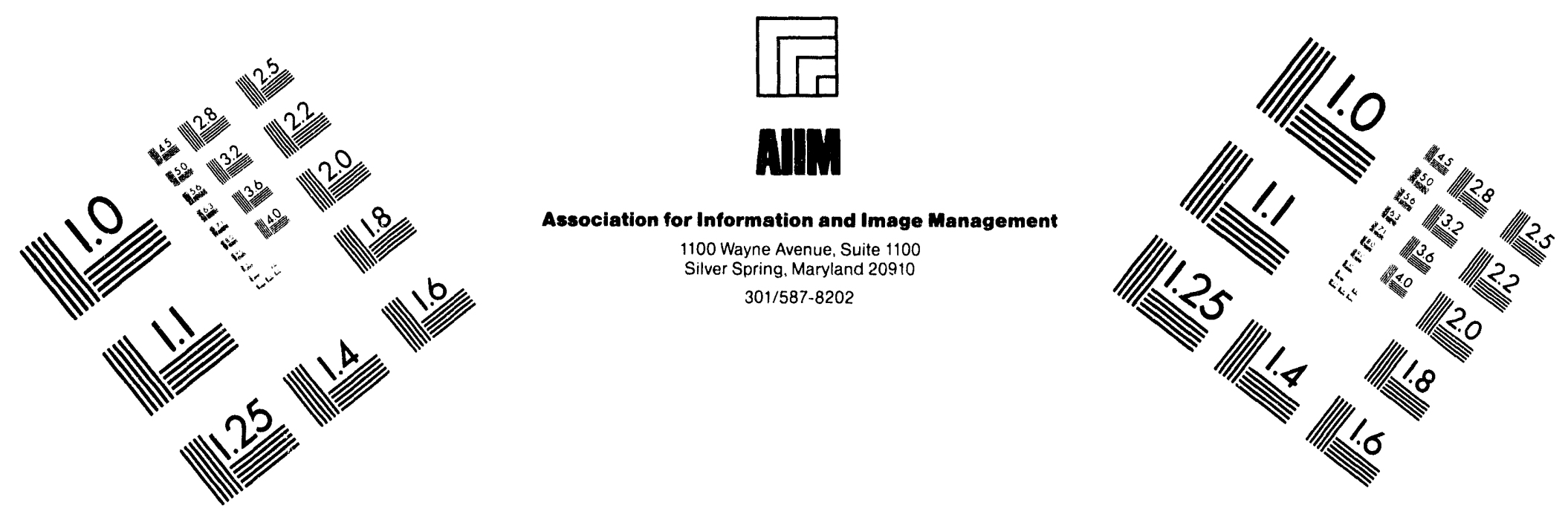

\title{
Centimeter
}

$\begin{array}{llllllllllllllll}1 & 2 & 3 & 4 & 5 & 6 & 7 & 8 & 9 & 10 & 11 & 12 & 13 & 14 & 15 & \mathrm{~mm}\end{array}$

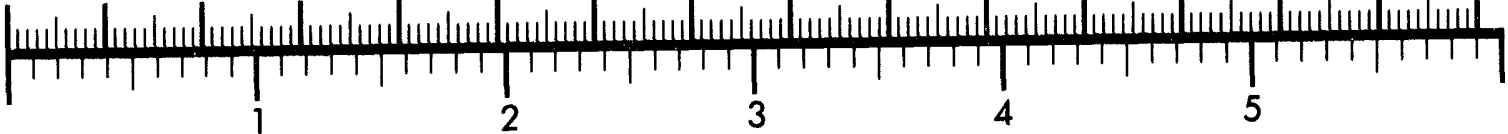
Inches
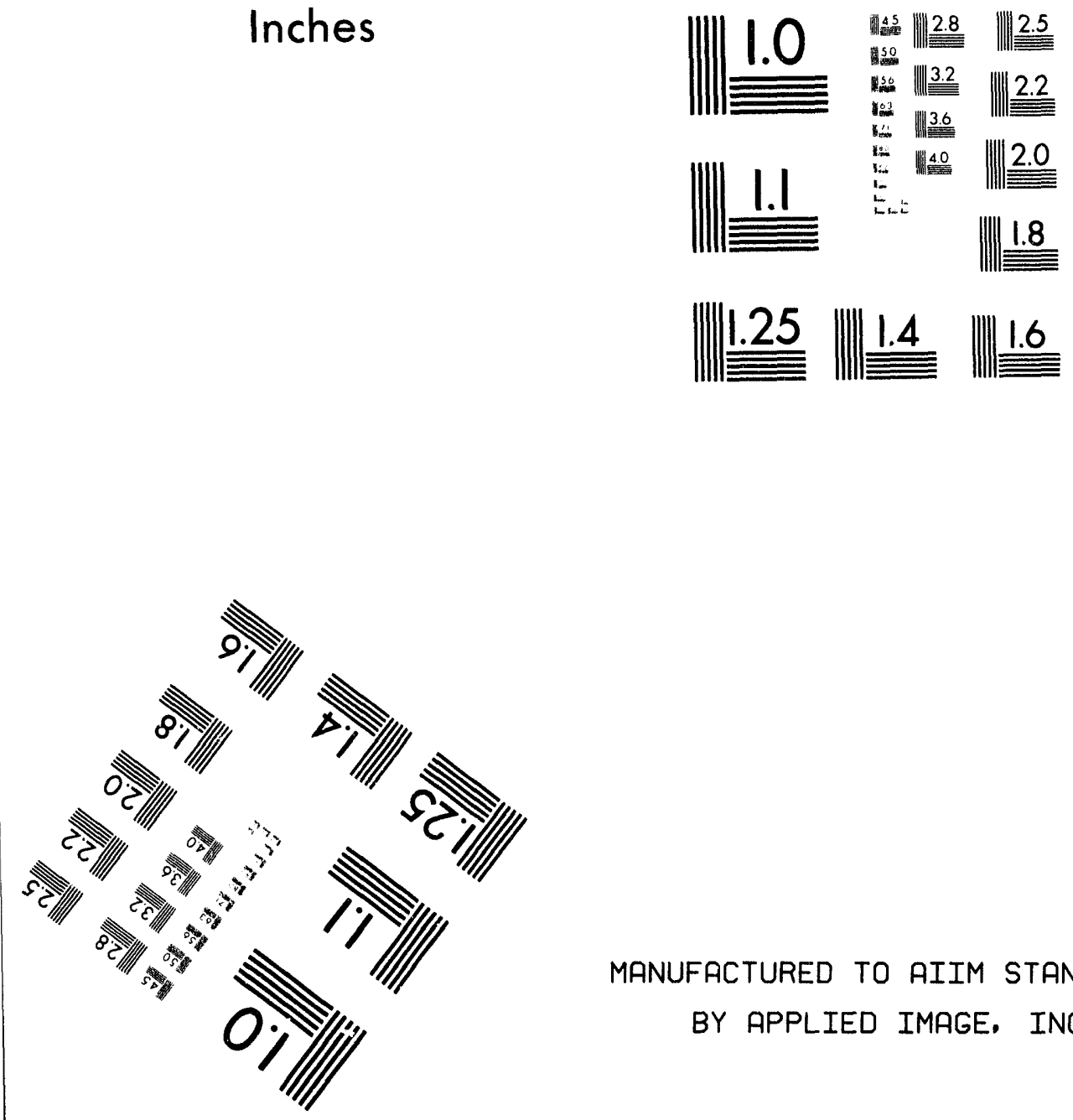

MANUFACTURED TO AIIM STANDARDS BY APPLIED IMAGE, INC.

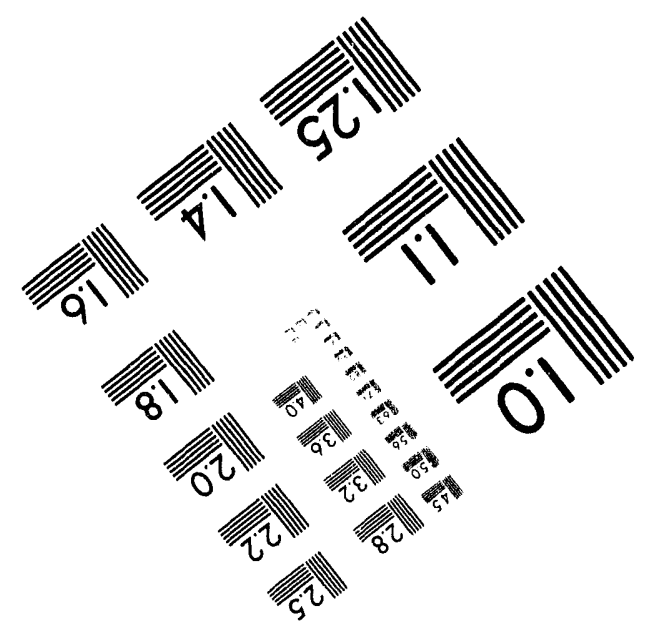



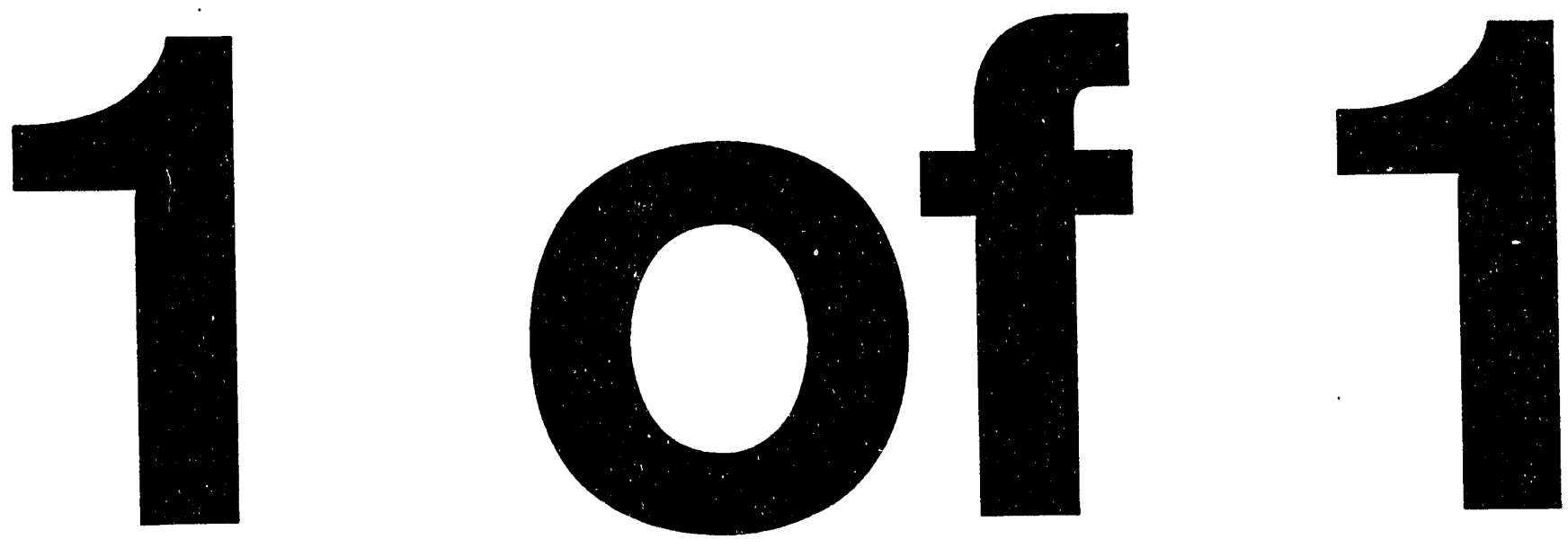

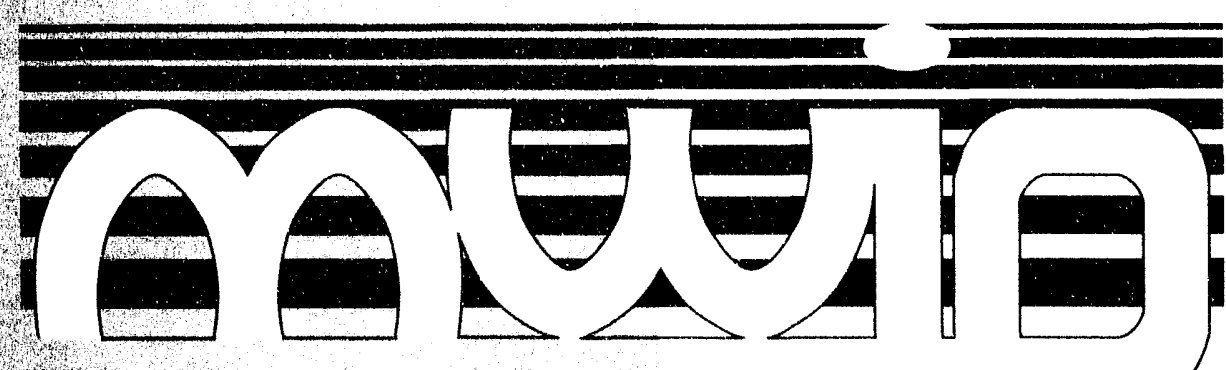

DOEIMWIP-25

LA-UR-94-928

\section{LIFE CYCLE COST ANALYSIS for the PLASMA ARC FURNACE}

Prepared for the Department of Energy-Office of Technology Development (EM-50), TTP Number AL-1212-19.

March 1994 
This report has been reproduced directly from the best available copy.

Available to DOE and DOE contractors from the Office of Scientific and Technical Information, P.O. Box 62, Oak Ridge, TN 37831; prices available from (615) 576-8401, FTS 626-8401.

Avaiiable to the public from the National Technical Information Service, U.S. Department of Commerce, 5285 Port Royal Rd., Springfield, VA 22161.

This report was prepared as an account of work sponsored by an agency of the United States Government. Neither the United States Government nor any agency thereof, nor any of their employees, makes any warranty, express or implied, or assumes any legal liability or responsibility for the accuracy, completeness, or usefulness of any information, apparatus, product, or process disclosed, or represents that its use would not intringe privately owned rights. Reference herein to any specific commercial product, process, or service by trade name, trademark, manufacturer, or otherwise, does not necessarily constitute or imply its endorsement, recommendation, or favoring by the United States Government or any agency thereof. The views and opinions of authors expressed herein do not necessarily state or reflect those of the United States Government or any agency thereof. 


\author{
LIFE CYCLE COST ANALYSIS \\ FOR THE \\ PLASMA ARC FURNACE
}

Peter Barnes-Smith

IT, Corp.

Albuquerque, NM

(505)262-8762

PRELIMINARY ISSUED FOR COMMENT

OCTOBER 20, 1993

\title{
DRAFT
}

\section{MASTER}




\title{
MIXED WASTE INTEgRATED PROGRAM
}

\author{
LIFE CYCLE COST COMPARISON \\ FOR THE \\ PLASMA ARC FURNACE STUDY
}

\section{EXECUTIVE SUMMARY}

\section{INTRODUCTION}

The Mixed Waste Integrated Program requested that the Systems Analysis Group investigate the cost effectiveness of using the Plasma Arc Furnace (PAF) module in place of specified thermal and final forms treatment equipment in the baseline Mixed Waste Treatment Project (MWTP) study as performed by Bechtel Corporation, September 1992.

The attached estimates are based on the process equipment and facilities cost data contained in the Bechtel study. The PAF process equipment and facilities cost data were developed using independent cost estimates for the equipment list provided by SAIC, Waste Management and Technology Division, in cooperation with the Pu!lution Prevention and Systems Analysis Group of the Oak Ridge National Laboratory, Chemical Technology Division.

\section{ESTIMATE METHODOLOGY}

In order to develop the total life cycle cost estimate comparison for this study, it was necessary to use a common base for comparison. Although it was felt that the Bechtel MWTP study did not fully reflect the optimum size for the thermal and final forms treatment equipment, it was the best available data at the time.

\section{Process Equipment}

In both the baseline cost evaluation and the alternative treatment cost evaluation, the equipment costs for the major process equipment items were taken from areas 800 and 900 of the MWTP estimate, and factored from the direct cost level to total capital cost level by using the stated cost multipliers as defined in the study estimate. In the alternative treatment evaluation, the total capital cost was developed by replacing or removing certain process equipment items in areas 800 and 900 , and applying the same multiplier factors to convert this direct cost to total capital cost.

\section{Support Operations Equipment}

The support operations equipment for areas 800 and 900 was factored from the MWTP cost estimate by the ratio of direct cost for the area process equipment against the direct cost for the 
total process equipment. This factor was intended to apportion the support operatic.is equipment to the user areas, in a direct ratio of process equipment cost.

\section{Building Facilities}

Using the MWTP cost summary for process buildings, the direct costs for building facilities for areas 800,900 and 1000 were factored according to their respective process floor areas versus the total process floor area. These direct costs were then treated to the same multipliers as the total building facilities cost to bring the direct cost to total capital cost.

The building facilities costs included the following:

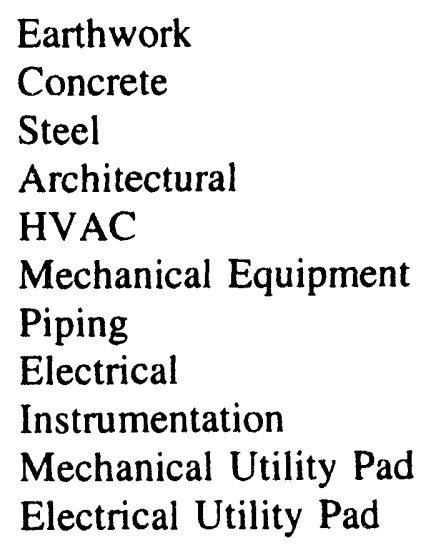

\section{Multiplier Factors}

The following multipliers were used to expand the direct cost level to total cost:

$\begin{array}{ll}\text { Process Controls } & 20 \% \text { of direct labor and materials } \\ \text { Indirect Field Costs } & \begin{array}{l}28 \% \text { of direct labor for material } \\ 75 \% \text { of direct labor for labor }\end{array} \\ \text { Contractor's OH\&P } & \begin{array}{l}5 \% \text { of materials and subcontracts } \\ 15 \% \text { of labor }\end{array} \\ \text { Construction Management } & 10 \% \text { of contractor's field cost } \\ \text { Architect/Engineer Costs } & 25 \% \text { of total field cost } \\ \text { Project Management } & 6 \% \text { of subtotal cost }\end{array}$


Contingency

Escalation
$25 \%$ of total cost

$4.4 \%$ per year

\section{Operations and Maintenance}

\section{Trial Burn}

A 2 week trial burn period was estimated for the thermal process equipment in order to establish emission parameters, and to determine the optimum processing rate for the equipment.

\section{$\underline{\text { Startup Cost }}$}

Based on the Waste Management Facilities Cost Information Report produced by Idaho National Engineering Laboratory, October 1992, a factor of $100 \%$ of the annual operating cost was used for the cost of starting up the process equipment. This factor includes all temporary equipment and utilities, consumable supplies and labor to establish the operability of the process.

\section{Annual Operating Cost}

Based on the MWTP study, the annual operating cycle was stated to be 31 weeks per year at 24 hours per day. This varies from most processing facilities which operate at 24 hours per day for 50 weeks per year. We have therefore extended the working year to 50 weeks to conform to industry standards. Operating cost includes direct and indirect labor and material, consumable supplies, utilities, and miscellaneous supplies including personnel protection.

\section{Annual Maintenance Cost}

Based on the Waste Management facilities Cost Information report, the cost of annual maintenance was factored at $17 \%$ for labor, $7 \%$ for materials, and $1 \%$ for supervision \& expenses against the fixed equipment cost.

\section{Annual Waste Disposal Cost}

Based on information received for disposal at Chemnuclear, Barnwell, South Carolina, and transportation from a generic central US facility, the cost of sampling, transport and disposal was calculated for the output from the MWTP flowsheet of $16,953 \mathrm{Kg} /$ week, over the 50 week operating period. 


\section{Contingency}

Based on the level of estimate development, a contingency of $25 \%$ of all costs excluding fixed capital was added to the total life cycle cost. This amount is intended to reflect the confidence of the scope, design and cost data used in this study.

\section{Discounted Cash Outflow}

The discounted cash outflow table is intended to normalize the study by bringing all cost back to a common point of reference. The $3.7 \%$ Real Discount Rate is extrapolated from the Office of Management and Budget (OMB) Circular A-94, Dated October 29, 1992 (Appendix "C") which indicates the discount rates for Cost-Effectiveness, Lease Purchase, and related analysis. We have used the real discount rate as directed by the OMB circular which basically removes the effect of expected or actual inflation for cost-effectiveness analysis. We have also used the mid-year discount factor rather than the end-of-year discount factor as the cash outflows tend to occur in a steady stream throughout the project life cycle.

\section{ASSUMPTIONS AND EXCLUSIONS}

The cost effectiveness analysis is based on the following assumptions:

The cost data presented in the Bechtel MWTP study had been checked for reasonableness and accuracy within the range of a conceptual study estimate.

It is assumed for life cycle comparison that although the construction cost will be averaged over a minimum 5 year construction period, all capital construction costs occur in year "0".

An allowance of $20 \%$ of the fixed equipment cost is required every 5 years for replacement of major process equipment items not covered by the annual maintenance account.

The building facilities size and cost data is within the historic range of accuracy for a mixed low level waste treatment facility for the DOE complex.

The capital cost multiplier factors are within the standard American Association of Cost Engineers range of multipliers for capital cost development for process and utility industries.

The cost estimate has centered on the Thermal Treatment (Area 800) and the Final Forms Treatment (Area 900 in order to reflect the major cost differential as determined by the size and cost of redundant equipment. No comparison has been made at the front end of the flowsheet between the baseline and alternative flowsheets to determine what, if any, 
impact to the life cycle cost is created by the changes in retrieval, handling, segregation and characterization that may occur when the PAF is introduced into the system.

The estimates for waste disposal are considered to be identical in both cases. No allowance has been made for any potential cost saving that may be realized from the ability of the alternative flowsheet to establish a volume reduction or to dispose of a vitrified or cast metal waste product at a lower cost disposal facility than that needed for the baseline flowsheet.

It is assumed that the Nevada test Site will not be available for long term storage and disposal of the final waste form, and that the Barnwell facility (or some other higher priced facility) will need to be used.

The waste characterization cost for the alternative flowsheet could increase significantly if it is necessary to establish the contents of every drum prior to incineration. It is therefore assumed that there will be no significant difference between the baseline and alternative characterization costs.

It is assumed that the plasma melting operation will not cause any migration from the low level classification of the final waste form, and that the waste form will still meet all disposal criteria for low level waste.

The life cycle cost analysis is sensitive to the availability and receiving costs of a permitted disposal facility. Any significant increase in the cost of transportation and disposal may result in any apparent advantage being minimized or reversed.

The removal of the glass melting facility in the final forms treatment area for the alternative flowsheet changes the criteria from that proposed in the SAIC draft flowsheet of April 02, 1993. It is our understanding that this furnace is not now required due to the anticipated ability of the slag from the plasma furnace to pass any tests for long term disposal criteria. Should this not be the case, then the capital cost for the alternative flowsheet would be increased significantly due to the approximately 3 to 1 ratio for slag to metal from the plasma furnace.

This study assumes the availability of sufficient low cost electric power, water, natural gas and rail/road transportation adjacent to the waste processing facility.

The following costs are excluded from this study:

Salvage costs for process and building equipment at the end of the useful project life cycle.

Decontamination and Decommissioning of process facilities and buildings. 
Down-time and consumable costs associated with electrode replacement for the PAF.

Land and land improvements.

Roads and utility lines to the plantsite.

Research and Development costs.

\section{CONCLUSION}

As indicated by the attached life cycle cost comparisons, the alternative flowsheet shows a distinct economic advantage over the baseline flowsheet.

The major differences are in process equipment and annual maintenance. Due to the fact that the annual maintenance is factored from the fixed capital equipment, the difference in the cost of the process equipment is the main driver for the large cost delta over the 20 year life cycle.

It should be noted however that this cost analysis is extremely sensitive to the cost of the fixed capital equipment, which is susceptible to large cost swings. Should it be necessary to add back the glass melting process into the final forms treatment, this would add approximately $\$ 11$ million to the fixed capital cost, which, when added to the annual maintenance cost would reflect in a $\$ 70$ million cost increase over the life cycle of the facility. It can be seen from this example that any major cost changes in the fixed capital cost will be multiplied by a factor of 6 at the total life cycle cost level.

In summary, we would comment that this study indicates that further engineering development work be carried out on the flowsheet definition for both cases. If we can improve the confidence of the flowsheet, capital cost estimates, and therefore the life cycle cost estimate, we would be better able to draw a more definitive conclusion from the data. At this point, there is sufficient uncertainty in both the baseline and alternative flowsheets to require that additional process development be carried out before we can prepare a more definitive cost analysis. 


\section{LIFE CYCLE COST COMPARISON}

\section{BETWEEN}

BASELINE AND ALTERNATIVE FLOWSHEETS 


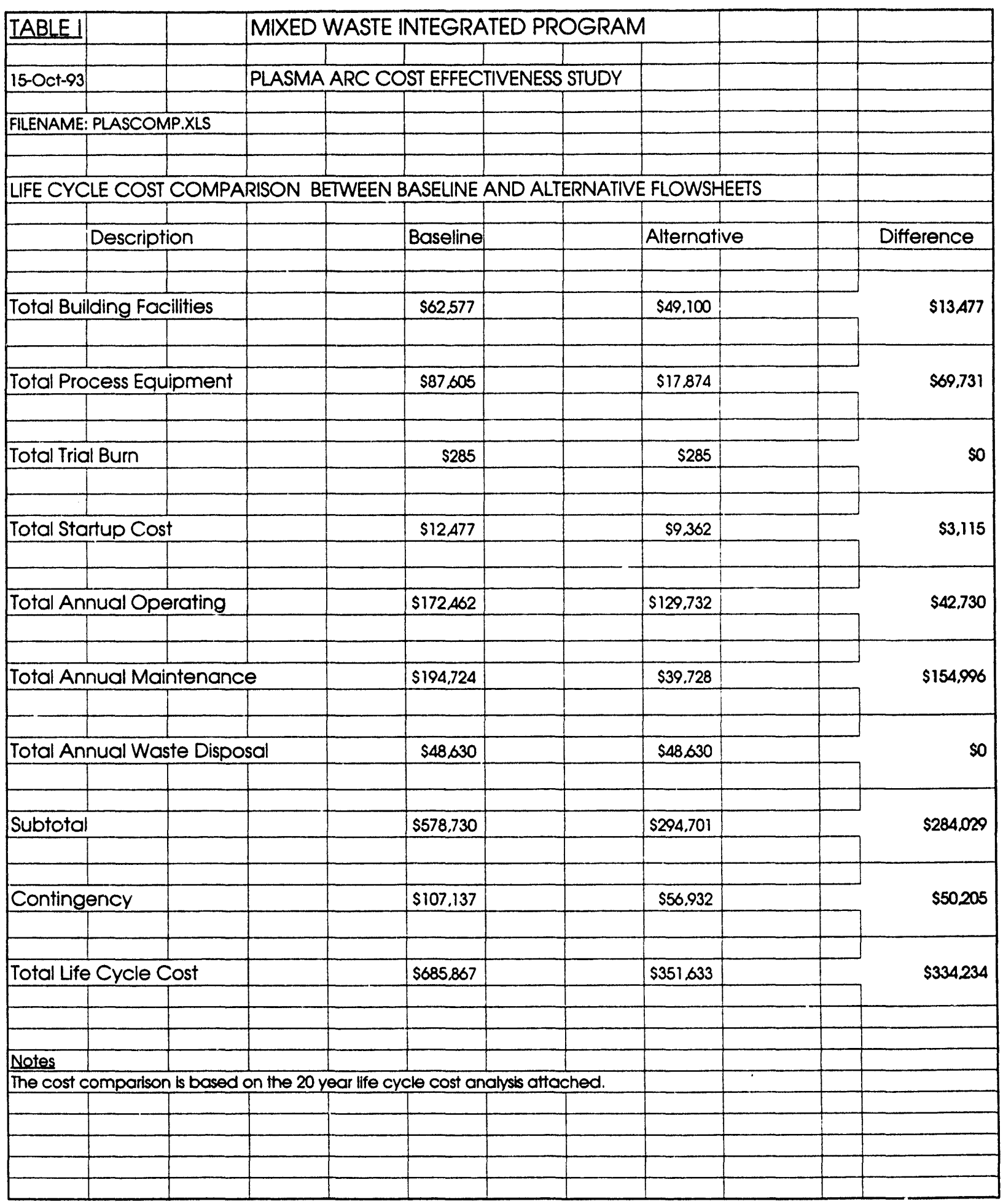


ALTERNATIVE THERMAL TREATMENT

LIFE CYCLE COST ESTIMATE 


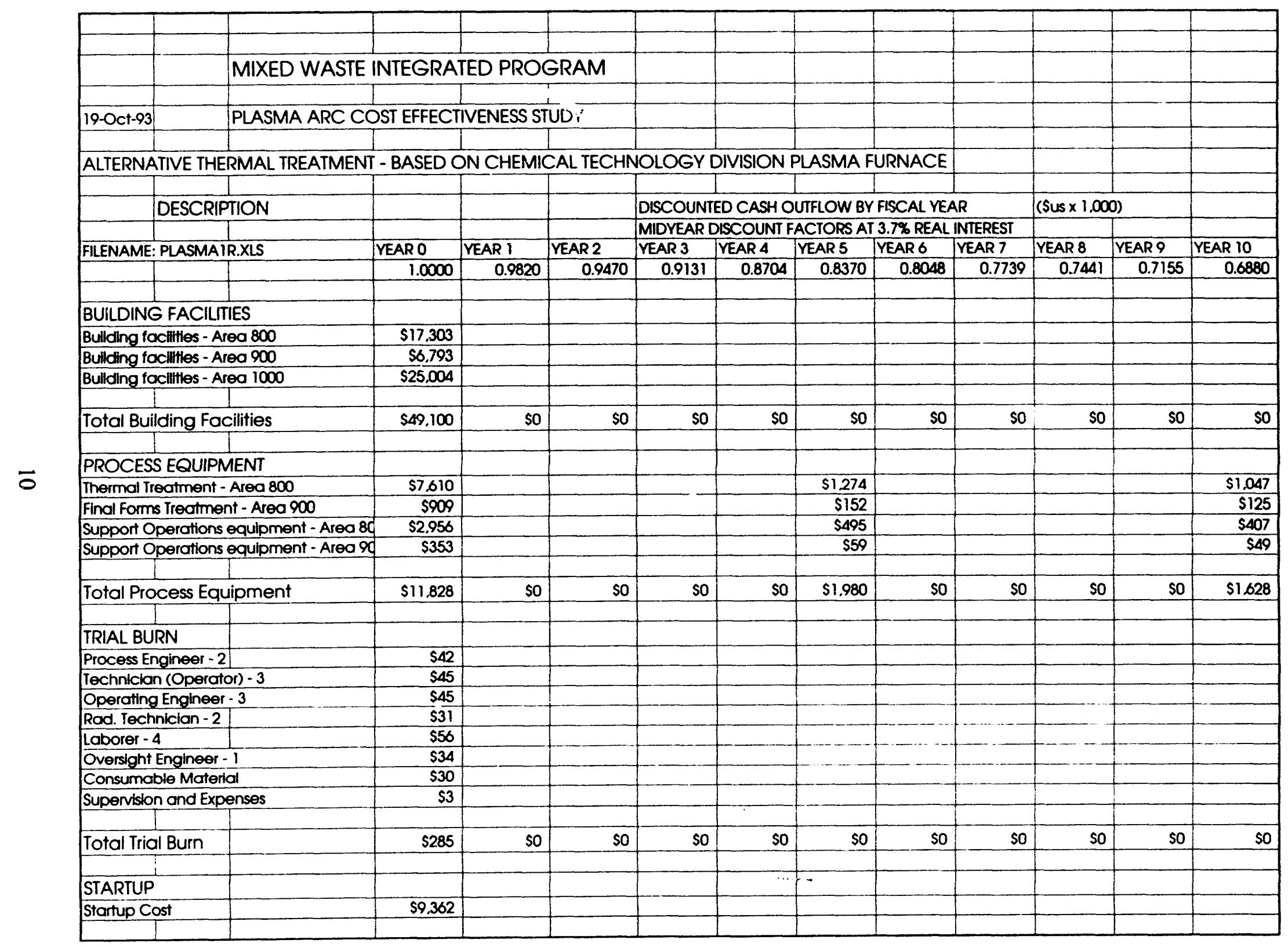




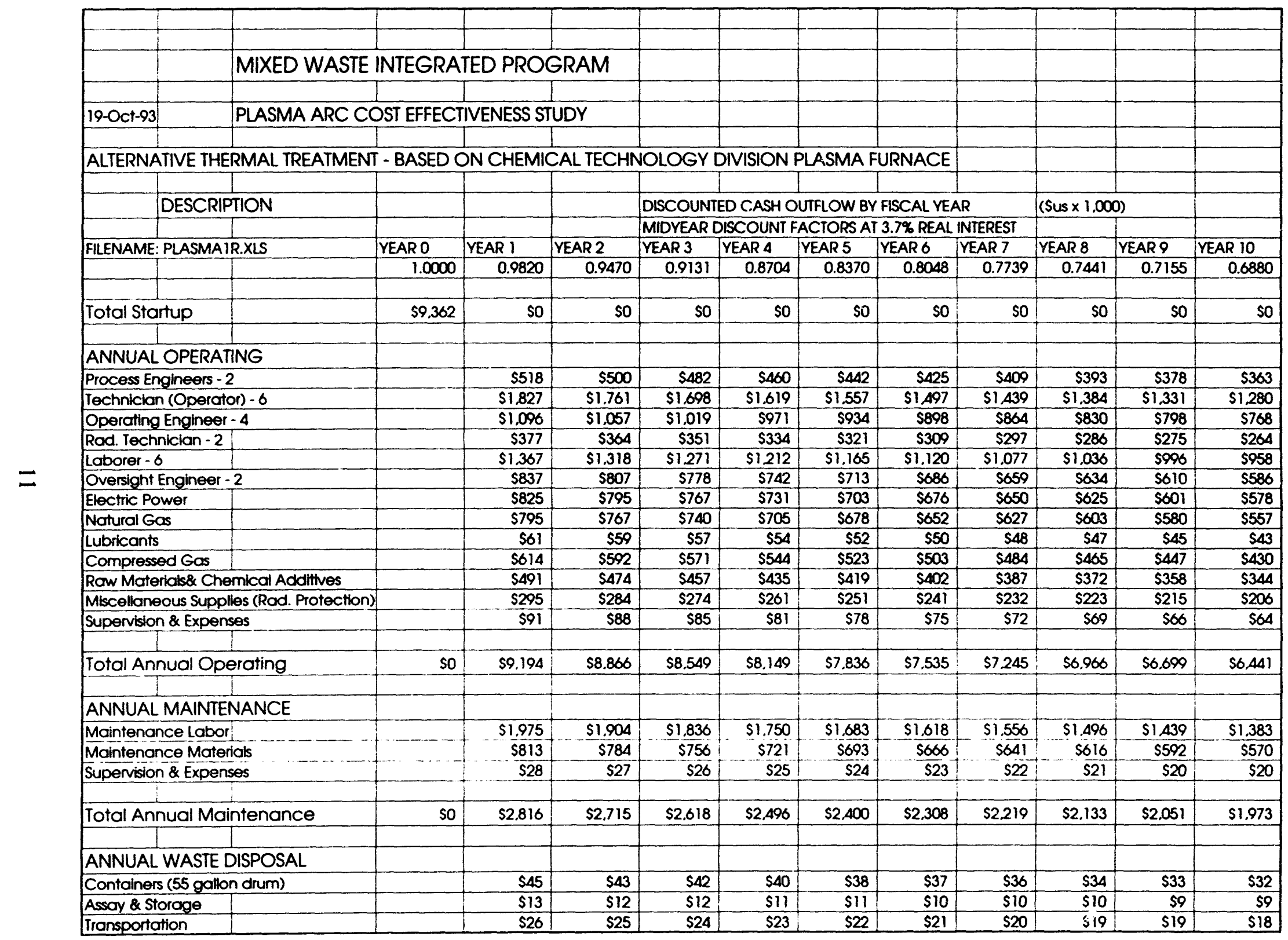




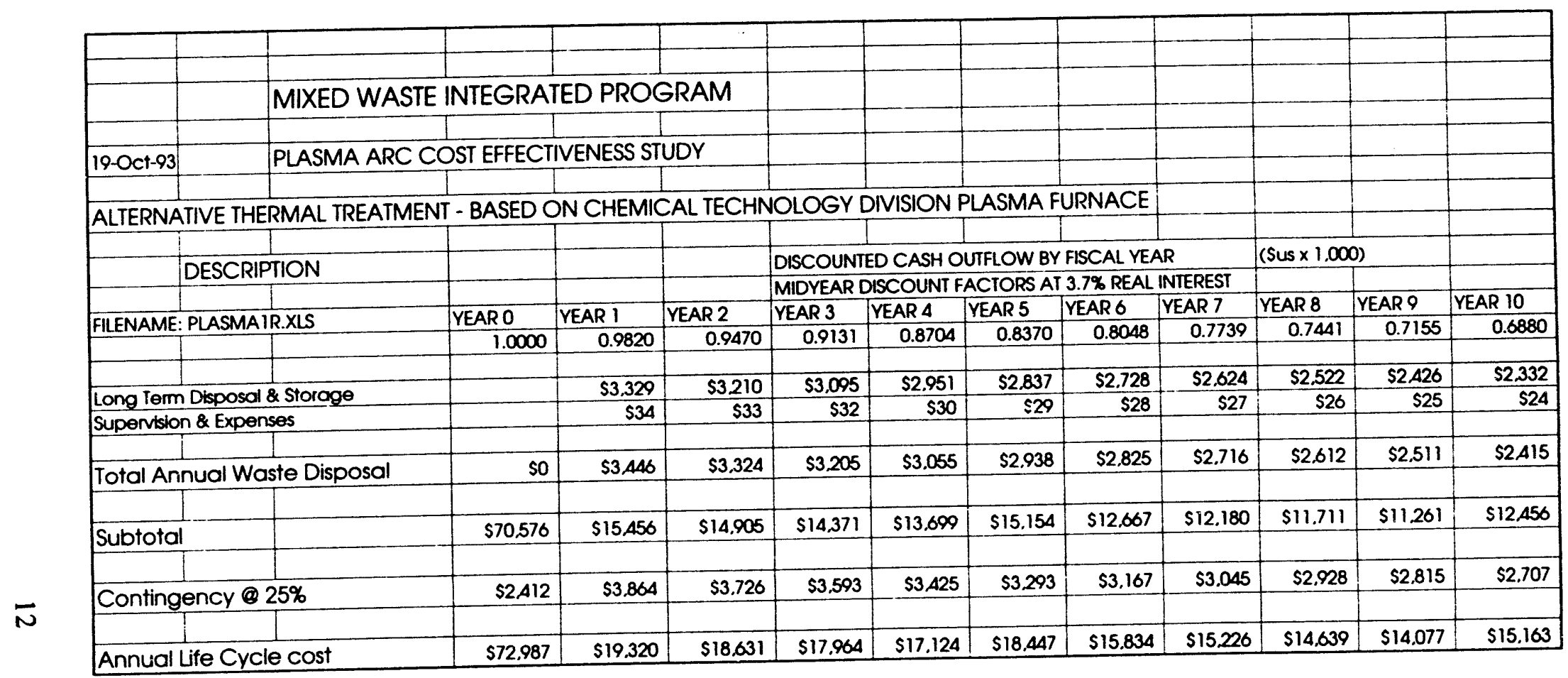




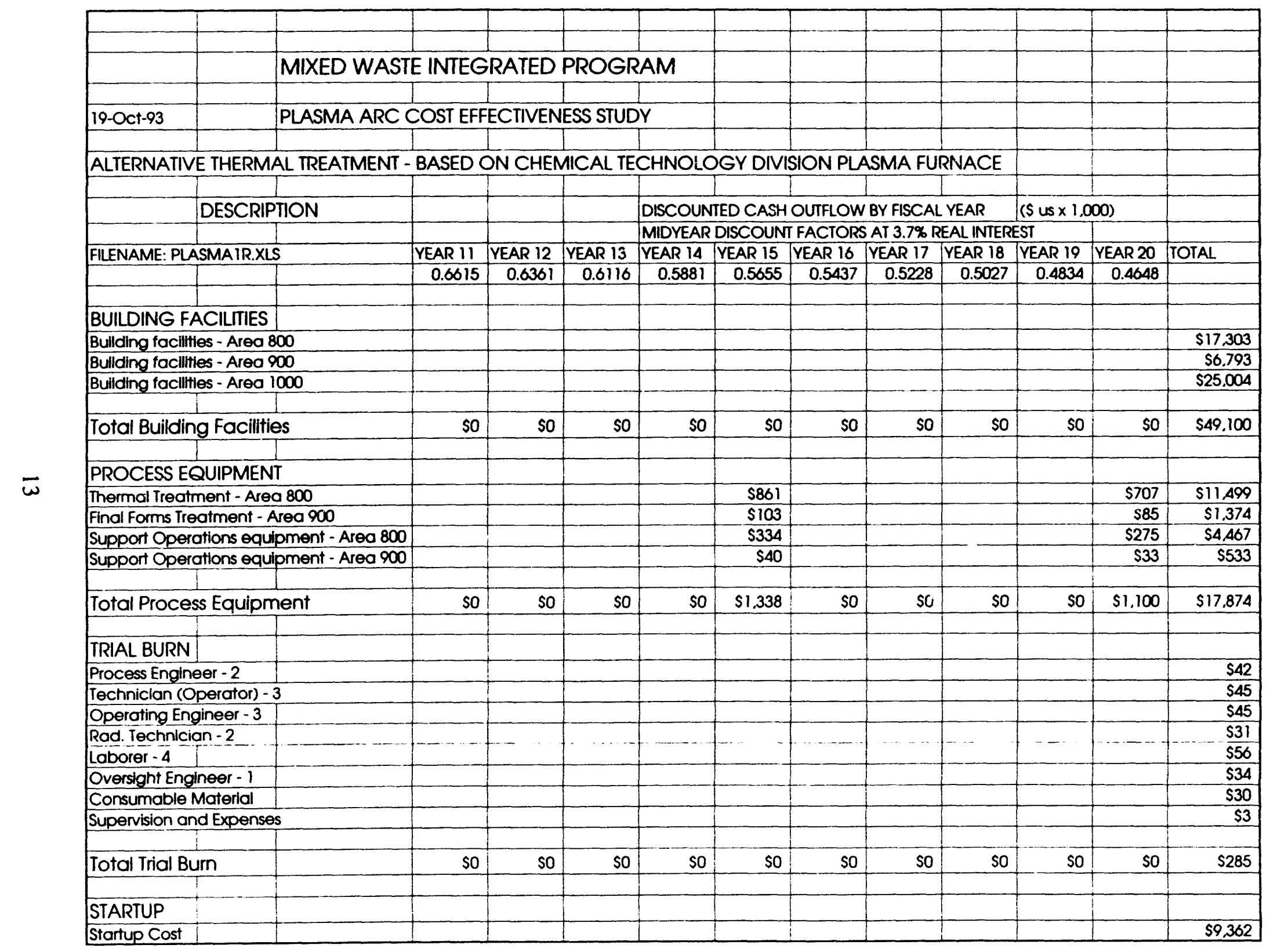




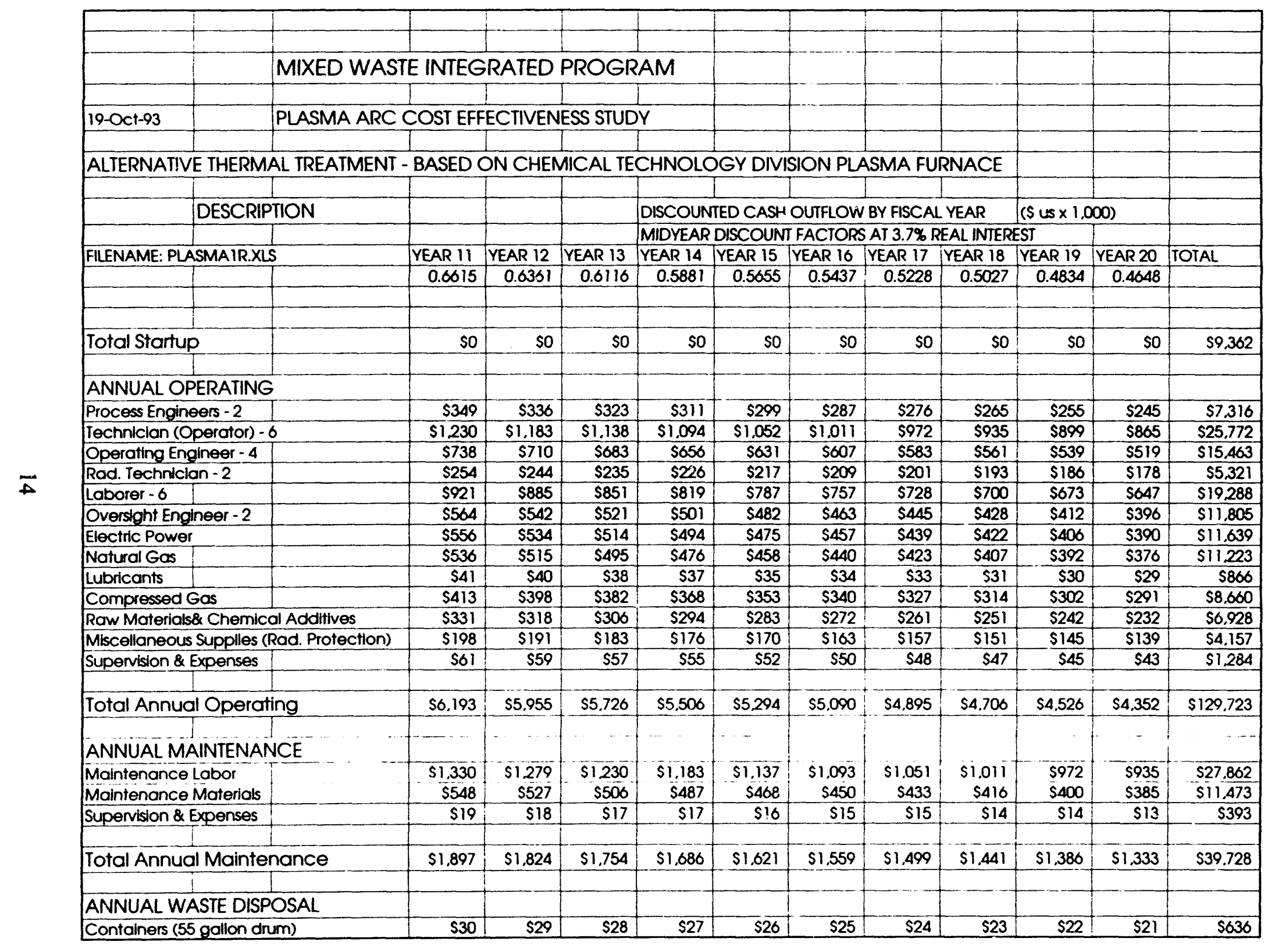




\begin{tabular}{|c|c|c|c|c|c|c|c|c|c|c|c|c|c|}
\hline \multirow{2}{*}{\multicolumn{14}{|c|}{ 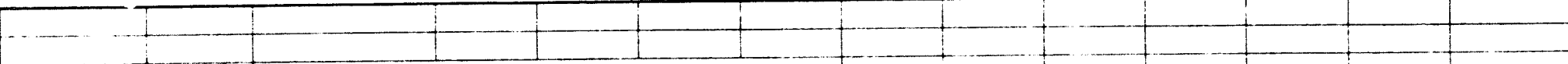 }} \\
\hline & & & & & & & & & & & & & \\
\hline & & \multicolumn{12}{|c|}{ MIXED WASTE INTEGRATED PROGRAM } \\
\hline $19-$ Oct-93 & & & & & & & & & & & & & \\
\hline & & \multicolumn{12}{|c|}{ PLASMA ARC COST EFFECTIVENESS STUDY } \\
\hline \multicolumn{14}{|c|}{ ALTERNATIVE THERMAL TREATMENT - BASED ON CHEMICAL TECHNOLOGY DIVISION PLASMA FURNACE } \\
\hline \multicolumn{14}{|c|}{\begin{tabular}{l|l|l|l|l|l|} 
ALTERNATIVE THERMAL TREATMENT - BASED ON CHEMICAL TECHNOLOGY DIVISION PLASMA FURNACE & \\
\end{tabular}} \\
\hline & \multicolumn{2}{|c|}{ DESCRIPTION } & & & & \multicolumn{7}{|c|}{\begin{tabular}{|l|l} 
DISCOUNTED CASH OUTFLOW BY FISCAL YEAR & $(S$ US $\times 1.000)$ \\
\end{tabular}} & \\
\hline & & & & & & \multicolumn{6}{|c|}{ MIDYEAR DISCOUNT FACTORS AT $3.7 \%$ REAL INTEREST } & & \multirow{3}{*}{ TOTAL } \\
\hline \multicolumn{3}{|c|}{ FILENAME: PLASMAIR.XLS } & YEAR 11 & YEAR 12 & YEAR 13 & YEAR 14 & YYEAR 15 & YEAR 16 & YEAR 17 & YEAR 18 & YYEAR 19 & YEAR 20 & \\
\hline 5 & & & 0.0615 & 0.6361 & 0.6116 & 0.5881 & 0.5655 & 0.5437 & 0.5228 & 0.5027 & 0.4834 & 0.4648 & \\
\hline \multicolumn{2}{|c|}{ Assay \& Storage } & & 59 & 58 & 58 & 58 & 57 & \$7 & s7 & $\$ 7$ & So & 56 & $\$ 180$ \\
\hline Transportat & & & $\$ 17$ & $\$ 17$ & $\$ 16$ & 515 & S15 & $\$ 14$ & 514 & si3 & 513 & $\$ 12$ & $\$ 360$ \\
\hline \multicolumn{3}{|c|}{ Long Term Disposal \& Storage } & 52.242 & $\$ 2.156$ & 52.073 & $\$ 1,994$ & $\$ 1.917$ & $\$ 1.843$ & $\$ 1.772$ & $\$ 1,704$ & 51.039 & $\$ 1,576$ & $\$ 46.972$ \\
\hline \multicolumn{2}{|c|}{ Supervision \& Expenses } & & $\$ 23$ & 522 & s21 & 520 & 520 & 519 & 518 & 517 & 517 & 516 & $\$ 481$ \\
\hline \multicolumn{3}{|c|}{ Total Annual Waste Disposal } & $\$ 2.322$ & $\$ 2232$ & $\$ 2.147$ & $\$ 2.064$ & $\$ 1,985$ & $\$ 1.908$ & $\$ 1.835$ & $\$ 1.764$ & $\$ 1.697$ & $\$ 1.031$ & $\$ 48,630$ \\
\hline & & & & & & 年, & , & & & & & & \\
\hline Subtotal & & & $\$ 10.411$ & $\$ 10.012$ & 59.626 & $\$ 9256$ & 510.238 & $\$ 8.557$ & 58.228 & $\$ 7.912$ & $\$ 7.608$ & $\$ 8,415$ & $\$ 294.701$ \\
\hline & & & & & & & & & & & & & \\
\hline \multicolumn{2}{|c|}{ Contingency @ 25\% } & & $\$ 2.603$ & $\$ 2.503$ & $\$ 2.407$ & $\$ 2,314$ & $\$ 2.225$ & $\$ 2.139$ & $\$ 2.057$ & $5 i .978$ & $\$ 1.902$ & $\$ 1.829$ & $\$ 56.932$ \\
\hline & & & & & & & & & & & & & \\
\hline \multicolumn{3}{|c|}{ Annual Life Cycle cost } & $\$ 13.014$ & $\$ 12.515$ & $\$ 12.033$ & $\$ 11,570$ & $\$ 12.463$ & $\$ 10,697$ & $\$ 10.285$ & 59.890 & $\$ 9.510$ & $\$ 10,244$ & $\$ 351,633$ \\
\hline
\end{tabular}




\section{BASELINE THERMAL TREATMENT}

COST ESTIMATE SUMMARY 


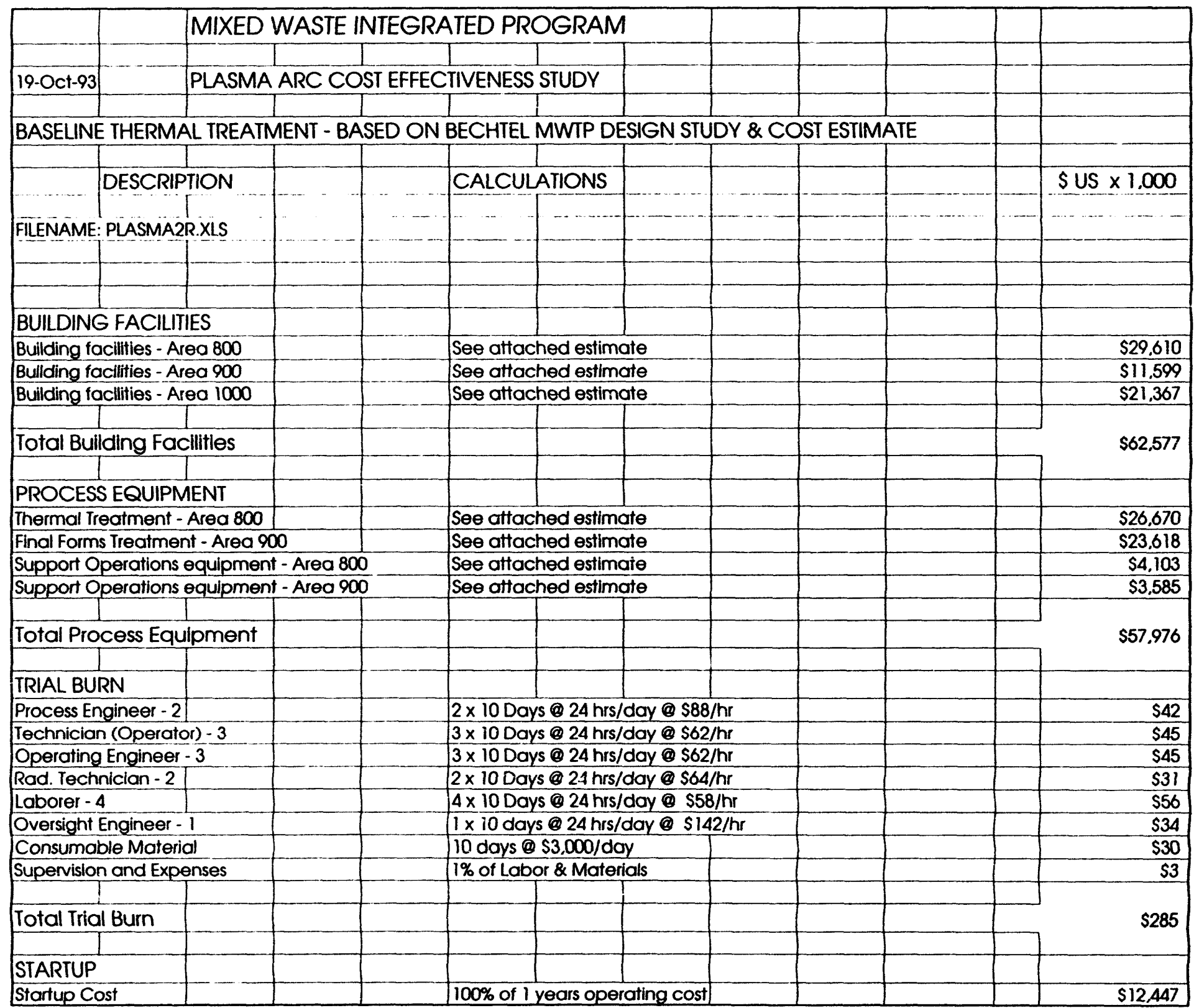




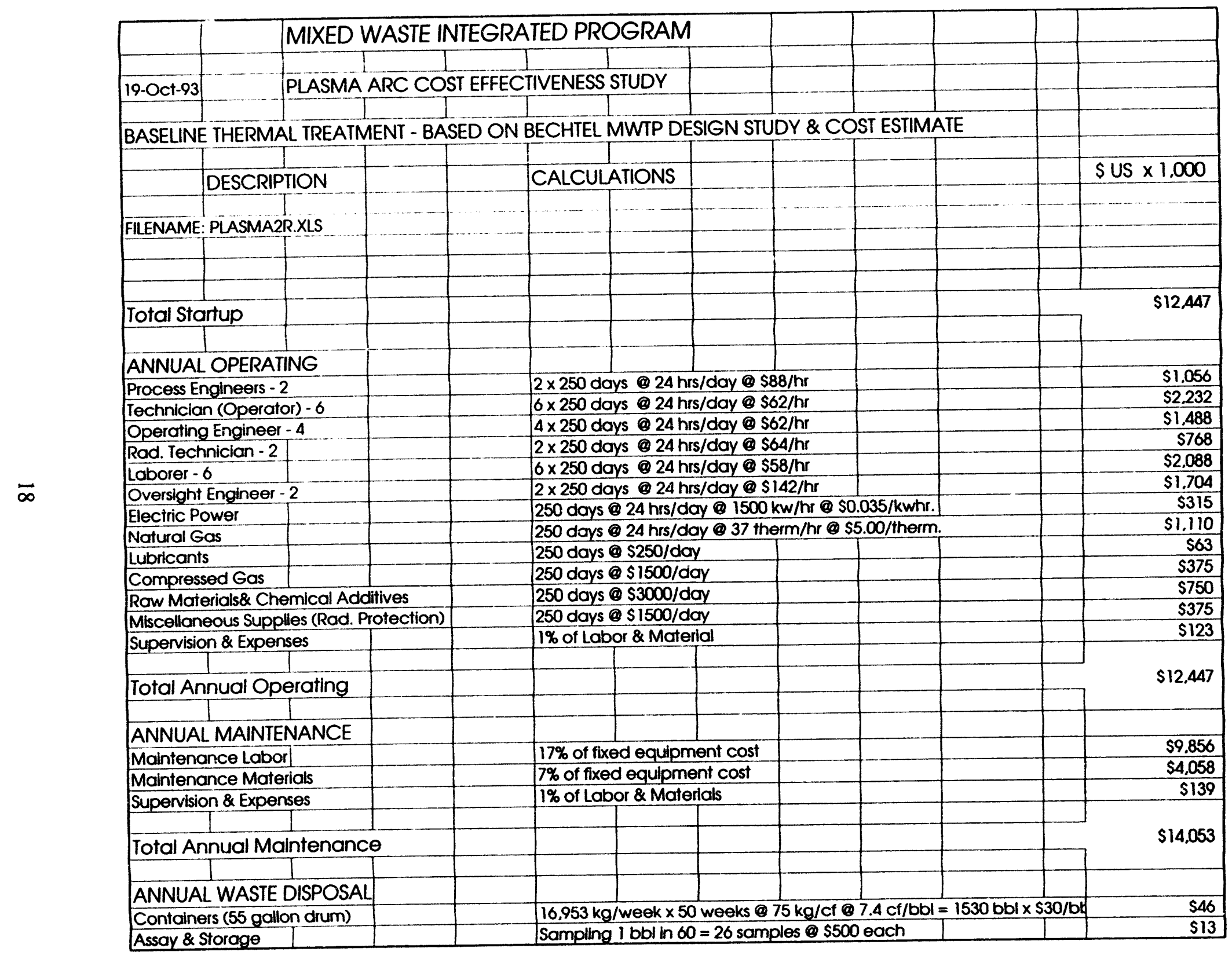




\begin{tabular}{|c|c|c|c|c|c|c|c|c|c|}
\hline & MIXED & WASTE & NTEGR, & ATED PROGRAM & & & & & \\
\hline & & & & & & & & & \\
\hline $19-$ Oct-93 & PLASMA & $A R C C C$ & ST EFFEC & CTIVENESS STUDY & & & & & \\
\hline & & & & & & & & & \\
\hline BASELINE & E THERMAL TREAT & AENT - BA & SSED ON & I BECHTEL MWTP DES & SIGN STUDY \& CC & STT ESTIM & ATE & & \\
\hline & & & & & & & & & \\
\hline & DESCRIPTION & & & CALCULATIONS & & & & & SUS $\times 1,000$ \\
\hline & & & & & & & & & \\
\hline FILENAME: & PLASMA2R.XLS & & & & & & & & \\
\hline & & & & & & & & & \\
\hline & & & & & & & & & \\
\hline Iransponto & ation & & & $1530 \mathrm{bbls} @ \mathrm{~s} / 7 \mathrm{each}$ & & & & & 526 \\
\hline Long Term & Disposal \& Storage & & & $11,300 \mathrm{cf} @ \$ 300 / \mathrm{cf}$ & & & & & $\$ 3.390$ \\
\hline Supervisior & $n$ \& Expenses & & & $1 \%$ of waste disposal & cost & & & & $\$ 35$ \\
\hline & & & & & & & & & \\
\hline Total Anr & nual Waste Dispc & & & & & & & & $\$ 3,510$ \\
\hline & & & & & & & & & \\
\hline & & & & & & & & & \\
\hline & & & & & & & & & \\
\hline & & & & & & & & & \\
\hline & & & & & & & & & \\
\hline & & & & & & & & & \\
\hline & & & & & & & & & \\
\hline
\end{tabular}


BASELINE THERMAL TREATMENT

\section{LIFE CYCLE COST ESTIMATE}




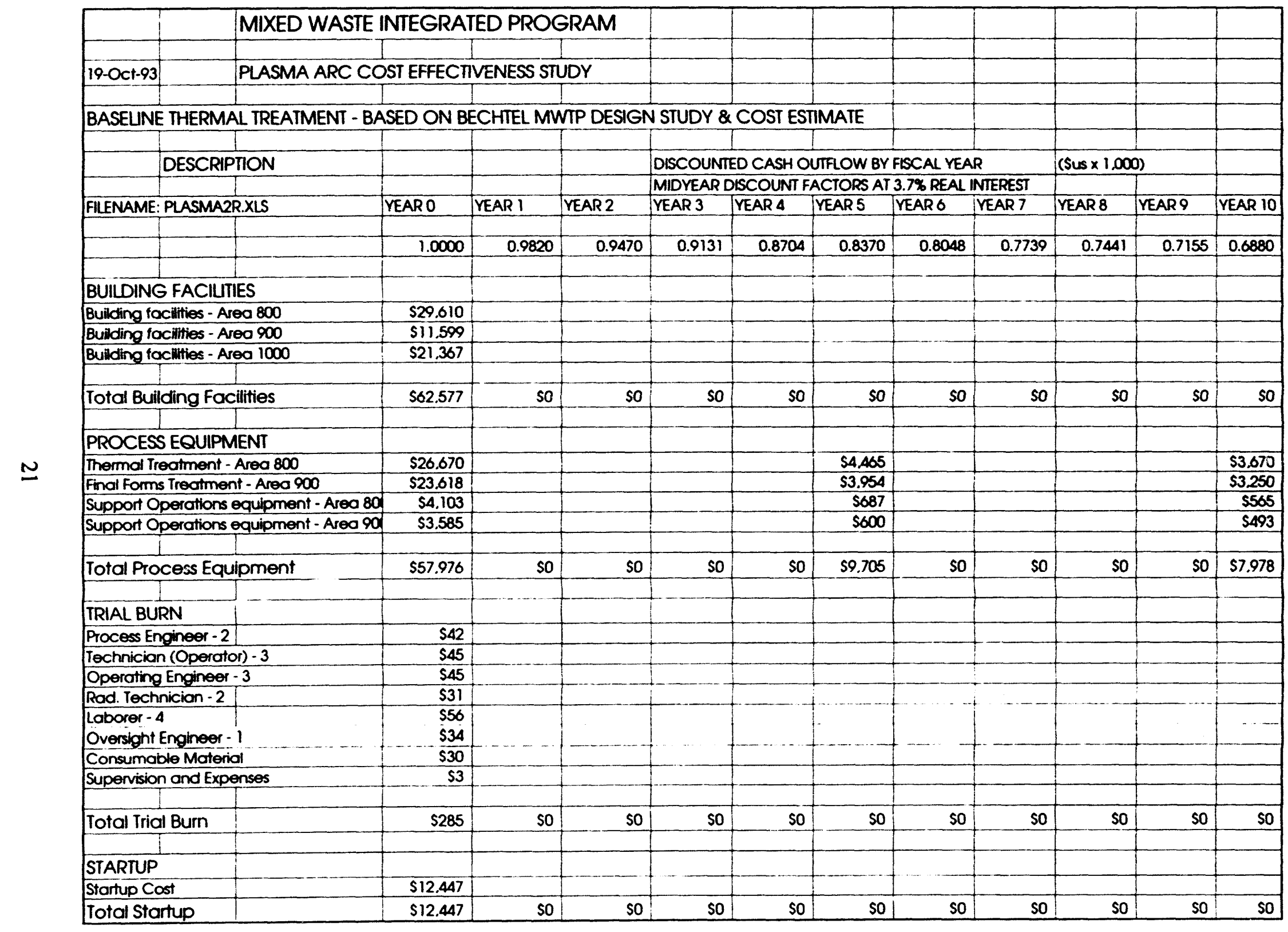




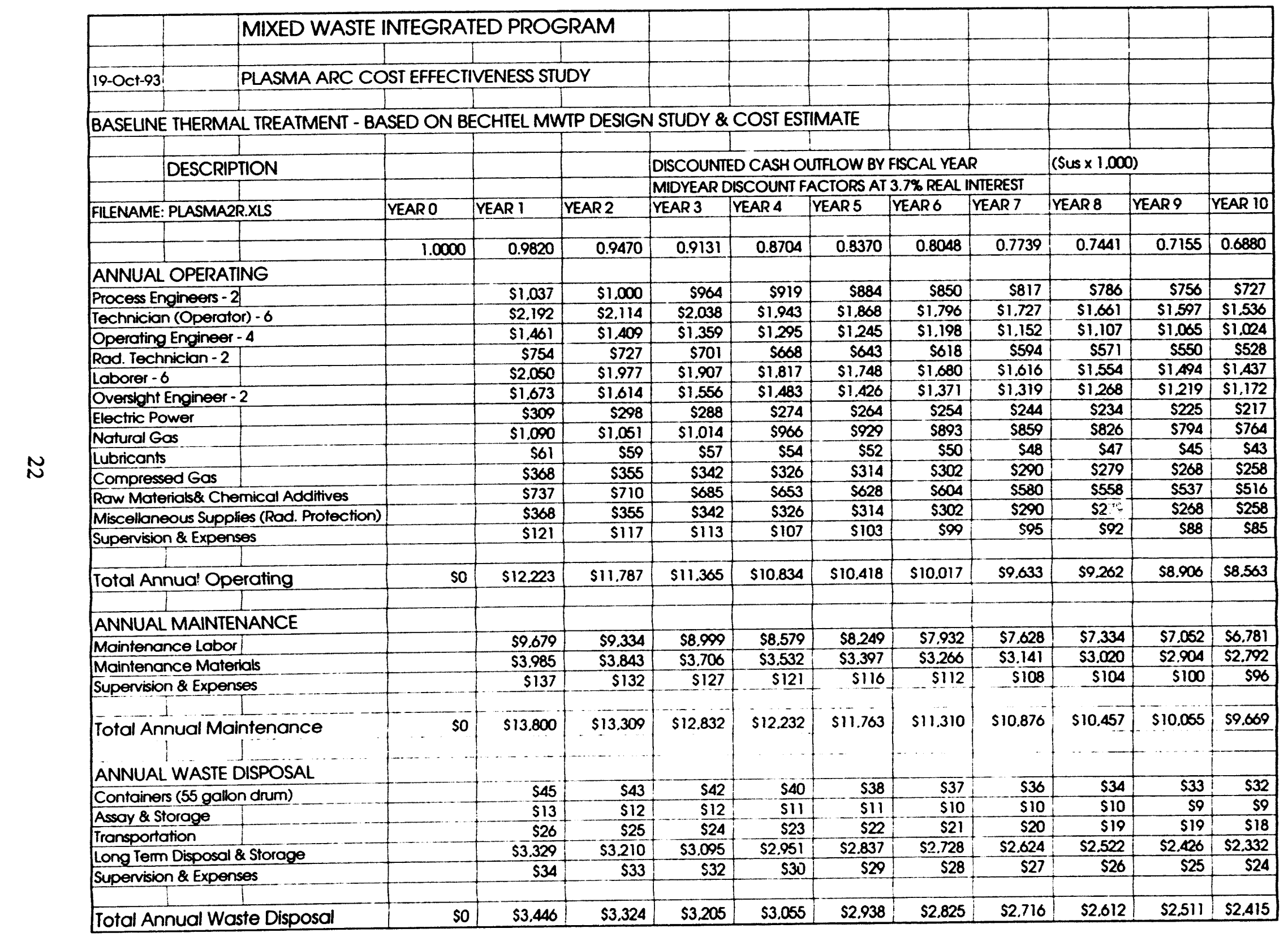




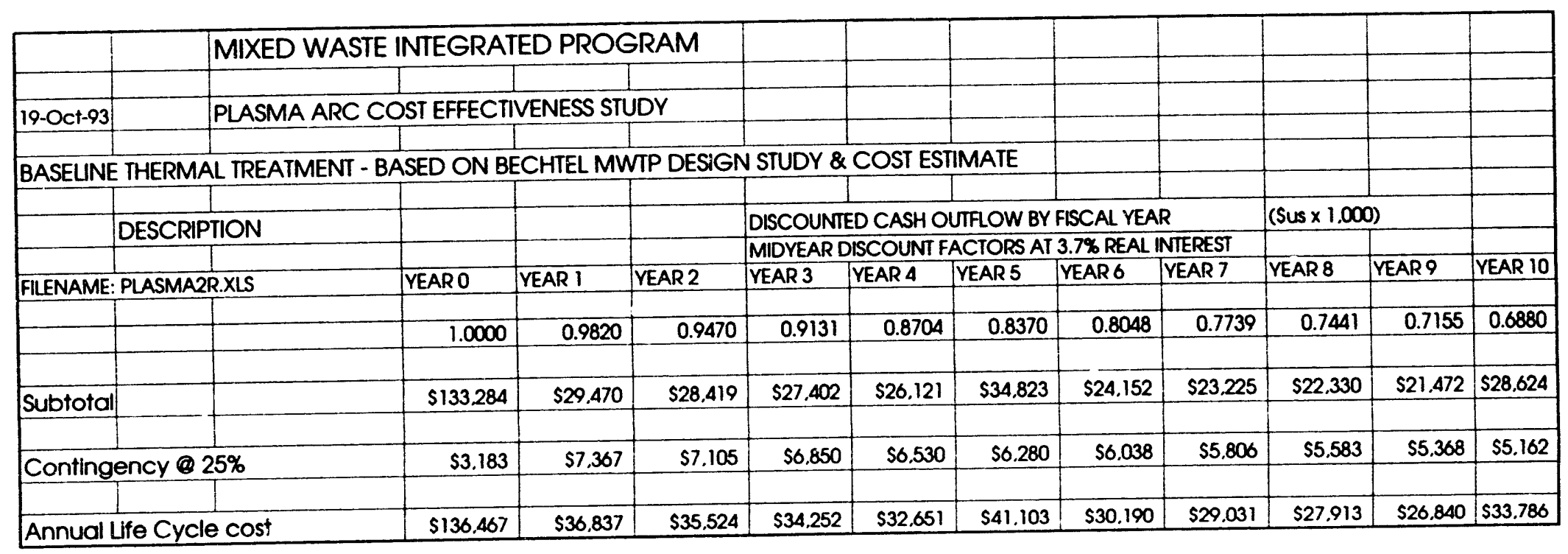




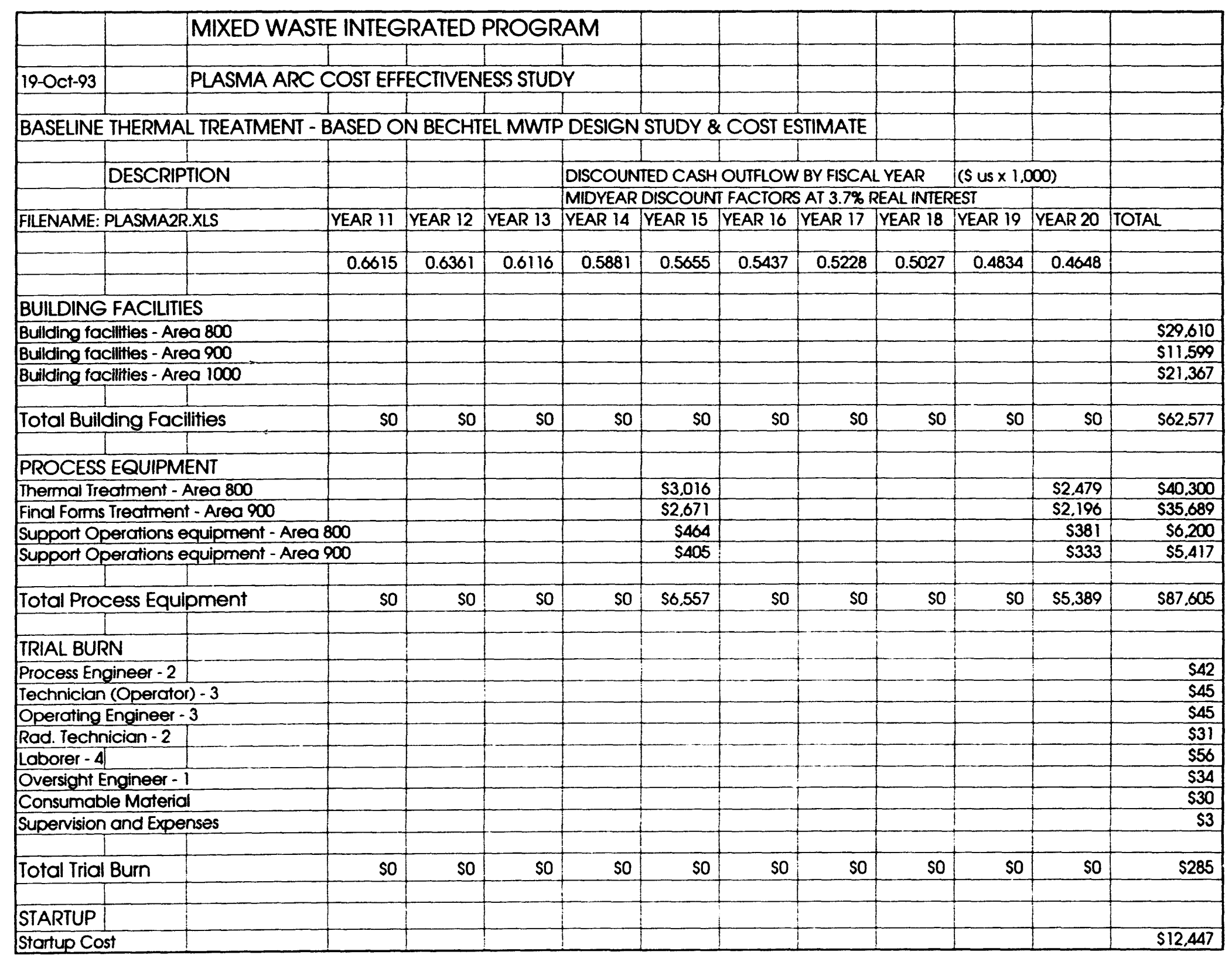




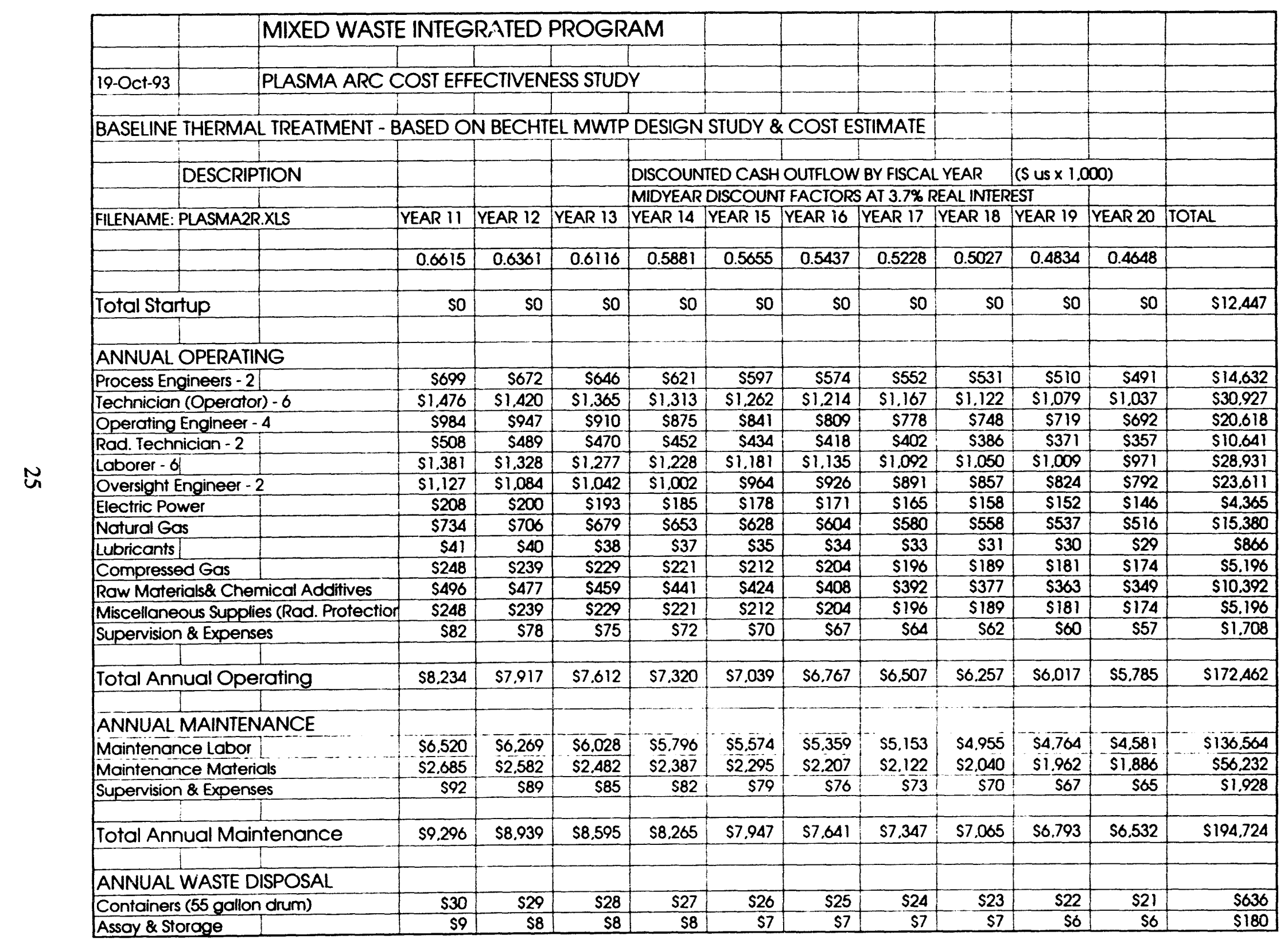




\begin{tabular}{|c|c|c|c|c|c|c|c|c|c|c|c|c|}
\hline & \multicolumn{5}{|c|}{ MIXED WASTE INTEGRATED PROGRAM } & & & & & & & \\
\hline \multirow{3}{*}{$19-0 c t-93$} & & & & & & & & & & & & \\
\hline & \multirow{2}{*}{\multicolumn{5}{|c|}{ PLASMA ARC COST EFFECTIVENESS STUDY }} & & & & & & & \\
\hline & + & & $\square$ & $I$ & & & & & & & & \\
\hline \multicolumn{13}{|c|}{ BASELINE THERMAL TREATMENT - BASED ON BECHTEL MWTP DESIGN STUDY \& COST ESTIMATE } \\
\hline & & & & & & & & & & & & \\
\hline & DESCRIPTION & & & & \multicolumn{5}{|c|}{ DISCOUNTED CASH OUTFLOW BY FISCAL YEAR } & \multicolumn{2}{|c|}{ (s us $\times 1.000)$} & \\
\hline & & & & & \multicolumn{6}{|c|}{ MIDYEAR DISCOUNT FACTORS AT $3.7 \%$ REAL INTEREST } & & \\
\hline \multicolumn{2}{|c|}{ FLLNAME: PLASMA2R.XLS } & YEAR II & YEAR 12 & YEAR 13 & YEAR 14 & YEAR 15 & YEAR 16 & YEAR 17 & YEAR 18 & YYEAR 19 & YEAR 20 & TOTAL \\
\hline & & & & & & & & & & & & \\
\hline & & 0.0615 & 0.6361 & 0.6116 & 0.5881 & 0.5655 & 0.5437 & 0.5228 & 0.5027 & 0.4834 & 0.4648 & \\
\hline Transportati & & $\$ 17$ & S17 & $\$ 16$ & $\$ 15$ & $\$ 15$ & $\$ 14$ & S14 & 513 & $\$ 13$ & $\$ 12$ & $\$ 360$ \\
\hline \multirow{2}{*}{\multicolumn{2}{|c|}{\begin{tabular}{|l|} 
Long Term Disposal \& Storoge \\
Supervision \& Expenses \\
\end{tabular}}} & $\$ 2.242$ & $\$ 2.156$ & $\$ 2,073$ & $\$ 1.994$ & $\$ 1.917$ & $\$ 1.843$ & $\$ 1.772$ & 51.704 & 51.639 & $\$ 1.576$ & $\$ 46.972$ \\
\hline & & $\$ 23$ & $\$ 22$ & $\$ 21$ & 520 & $\$ 20$ & $\$ 19$ & 518 & $\$ 17$ & 517 & 516 & 5481 \\
\hline & & & & & & & & & & & & \\
\hline \multicolumn{2}{|c|}{ Total Annual Waste Disposal } & $\$ 2,322$ & $\$ 2.232$ & $\$ 2.147$ & $\$ 2,064$ & $\$ 1.985$ & $\$ 1.908$ & $\$ 1.835$ & $\$ 1.764$ & $\$ 1.697$ & $\$ 1,631$ & $\$ 48.630$ \\
\hline & & & & & & & & & & & & \\
\hline Subtotal & & $\$ 19.851$ & $\$ 19.089$ & $\$ 18.354$ & $\$ 17.649$ & $\$ 23.528$ & $\$ 16.316$ & $\$ 15.689$ & $\$ 15.086$ & $\$ 14,507$ & $\$ 19,338$ & $\$ 578.730$ \\
\hline & & & & & & & & & & & & \\
\hline \multicolumn{2}{|c|}{ Contingency@25\% } & $\$ 4.963$ & $\$ 4,772$ & $\$ 4,589$ & $\$ 4,412$ & $\$ 4.243$ & $\$ 4.079$ & $\$ 3.922$ & $\$ 3.771$ & $\$ 3.627$ & $\$ 3.487$ & 5107.137 \\
\hline & & & & & & & & & & & & \\
\hline \multicolumn{2}{|c|}{ Annual Life Cycle cost } & $\$ 24.814$ & $\$ 23.862$ & $\$ 22.943$ & $\$ 22,061$ & $\$ 27.770$ & $\mathbf{s 2 0 , 3 9 5}$ & $\$ 19,011$ & $\$ 18.857$ & $\$ 18.133$ & $\$ 22.825$ & 5685.867 \\
\hline & & & & & & & & & & & & \\
\hline
\end{tabular}


BASELINE THERMAL TREATMENT

CAPITAL COST ESTIMATE 


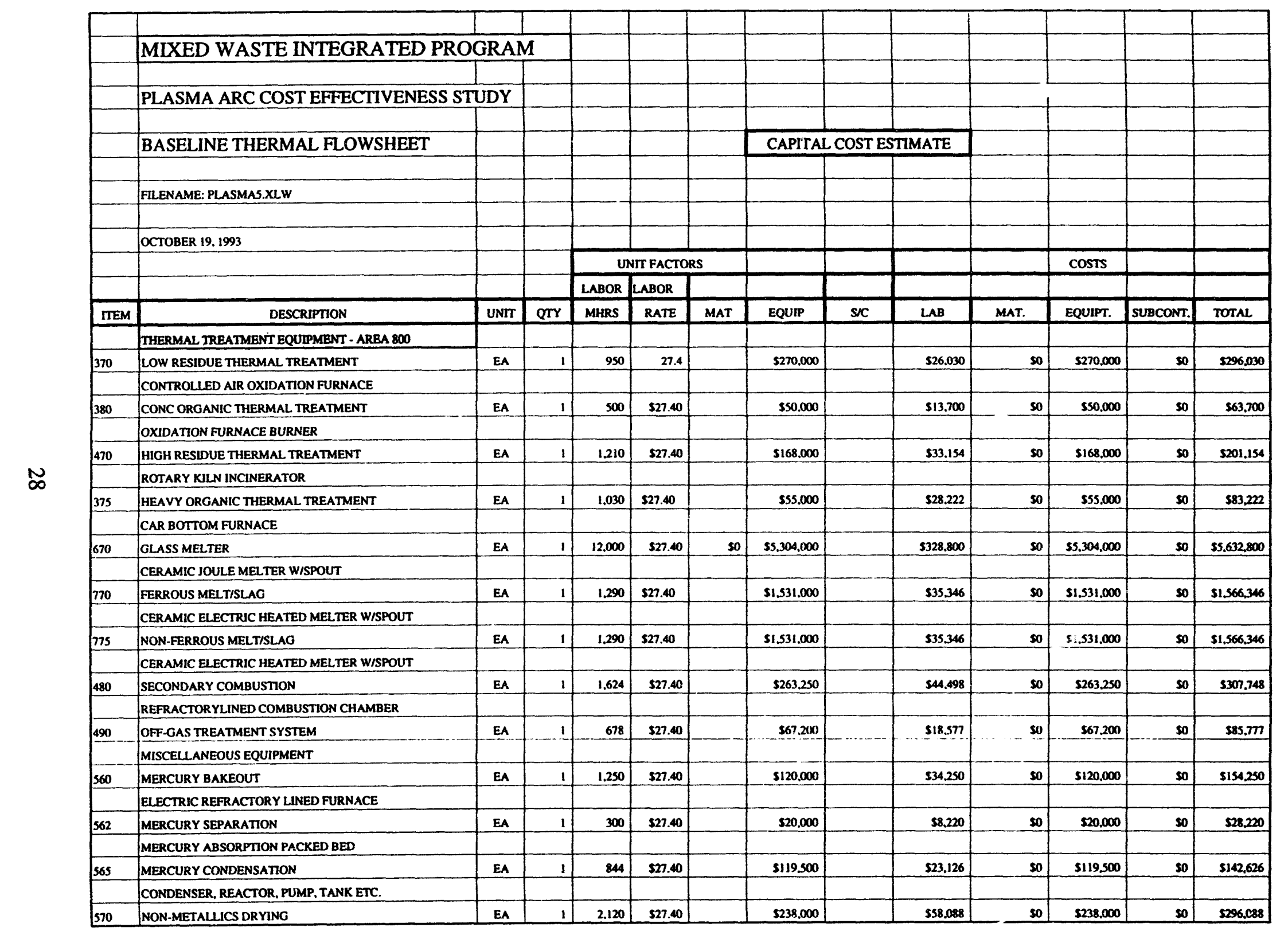




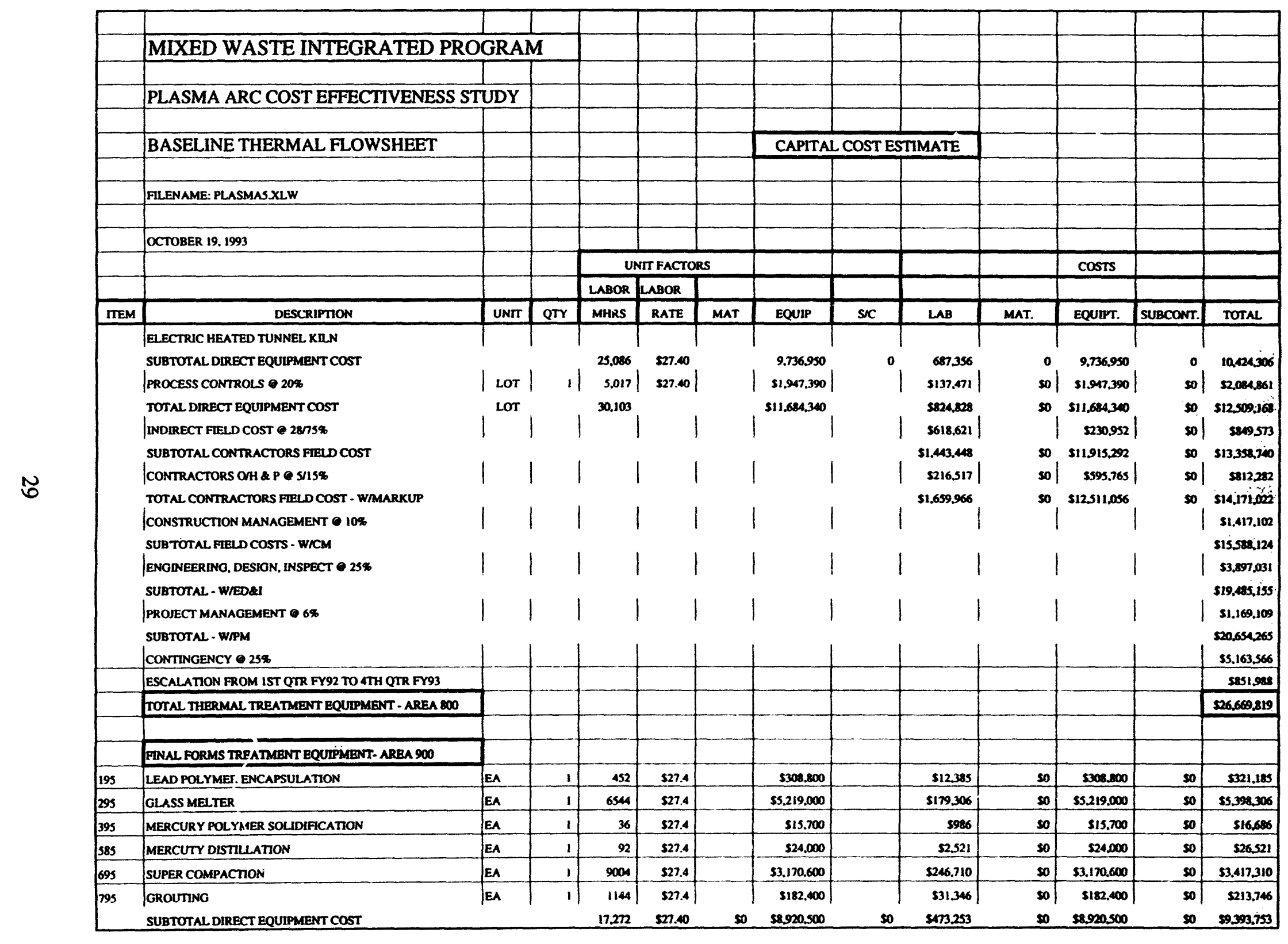




\begin{tabular}{|c|c|c|c|c|c|c|c|c|c|c|c|c|c|}
\hline & \multicolumn{3}{|c|}{ MIXED WASTE INTEGRATED PROGRAM } & & & & & & & & & & \\
\hline & & & & & & & & & & & & & \\
\hline & \multicolumn{2}{|l|}{ PLASMA ARC COST EFFECTIVENESS STUDY } & & & & & & & & & & & \\
\hline & & & & & & & & & & & & & \\
\hline & BASELINE THERMAL FLOWSHEET & & & & & & \multicolumn{3}{|c|}{ CAPITAL COST ESTIMATE } & & & & \\
\hline & & & & & & & & & & & & & \\
\hline & FLLENAME: PLASMAS:XLW & & & & & & & & & & & & \\
\hline & & & & & & & & & & & & & \\
\hline & OCTOBER 19.1993 & & & & & & & & & & & & \\
\hline & & & & \multicolumn{3}{|c|}{ UNTT FACTORS } & & & & & costs & & \\
\hline & & & & LABOR & LABOR & & & & & & & & \\
\hline ITEM & DESCRIPTION & UNT & QTY & MHRS & RATE & MAT & EQUIP & sec & LAB & MAT. & EQUIPT. & subcont. & TOTAL \\
\hline & PROCESS CONTROLS 20\% & LOT & 1 & 3.454 & $\$ 22.40$ & & $\$ 1.784 .100$ & & 594.651 & so & 51.724 .100 & $\$ 0$ & S1.878.751 \\
\hline & $\begin{array}{l}\text { TOTAL DIRECT EQUIPMENT COST } \\
\text { |INDIRECT FELD COST } @ 2875 \%\end{array}$ & LOT & ! & | & 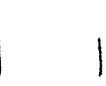 & & $\$ 10.704,600$ & & $\left|\begin{array}{l}\mathbf{s 5 6 7 . 9 0 3} \\
\mathbf{S 1 2 3 . 9 2 8}\end{array}\right|$ & so & $\begin{array}{l}\$ 10,201,600 \\
\$ 159.013\end{array}$ & 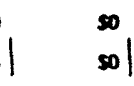 & $\begin{array}{r}511,272500 \\
5584,900\end{array}$ \\
\hline & SUBTOTAL CONTRACTORS FELD COST & & & & & & & & s993,831 & so & 510.863 .613 & so & $511.857,444$ \\
\hline & | CONTRACTORS OH \& P S/15\% & & 1 & & | & | & & & 5149.075 & so! & S543.18i & 501 & 5602.255 \\
\hline & TOTAL CONTRACTORS FIELD COST - WMARKUP & & & & & & & & $\$ 1.142 .906$ & so & $\$ 11,406,794$ & $\infty$ & 512.509 .609 \\
\hline & $\begin{array}{l}\text { CONSTRUCTION MANAGEMENT } 10 \% \\
\text { SUBTOTAL FELLD COSTS - W/CM }\end{array}$ & & & & 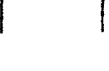 & & | & & & & & & $\begin{array}{r}\$ 1,251,970 \\
\$ 13,204,600\end{array}$ \\
\hline & |ENGNEERING. DESIGN, INSPECT $25 \%$ & 1 & | & 1 & 1 & 1 & 1 & & 1 & 1 & & 1 & | $\$ 3.451 .167$ \\
\hline & SUBTOTAL - W/ED\&I & & & & & & & & & & & & $\$ 17.255,836$ \\
\hline & |PROJECT MANAGEMENT @ 6\% & & 1 & 1 & | & 1 & | & & 1 & 1 & & | & $\mathbf{\$ 1 . 0 3 5 . 3 5 0}$ \\
\hline & $\begin{array}{l}\text { SUBTOTAL - W/PM } \\
\text { CONTINGENCY O 25\% }\end{array}$ & & & & & & & & & & & & $\begin{array}{r}\$ 18291,186 \\
\$ 4,572,797\end{array}$ \\
\hline & ESCALATION FROM IST QTR FY92 TO 4TH QTR FY93 & & & & & & & & & & & & 5754511 \\
\hline & FOTAL FNAL FORMS TREATMENT EQUIPMENT - ARRA 900 & & & & & & & & & & & & $223,618,494$ \\
\hline & & & & & & & & & & & & & \\
\hline & SUTPORT OPERATIONS EQUIPMENT - AREA 800 & & & & & & & & & & & & \\
\hline 100 & FEED AND SEGREGATION HANDLLNG & & & & & & & & so & 551.264 & & & 551,264 \\
\hline 280 & SECOND STAGE GAS HANDLING & & & & & & & & 57.004 & $\$ 142.400$ & & & siso.204 \\
\hline 290 & OFF-GAS CLEAN-UP & & & & & & & & $\$ 11.159$ & $\$ 127.733$ & & & $\$ 138,002$ \\
\hline 650 & ATMOSPHERIC PROTECTION & & & & & & & & 535.584 & 2226.416 & & & $\$ 262.000$ \\
\hline 900 & CONTANER DECONTAMINATION & & & & & & & & 535.428 & $\$ 210.166$ & & & 224594 \\
\hline 1100 & LOW LEVEL WASTE HANDLING & & & & & & & & 318.323 & $\$ 112.286$ & & & $\$ 130.609$ \\
\hline 1200 & PACKAGE ASSAY & & & & & & & & $\$ 11549$ & 5365.968 & & & sonsin \\
\hline 1300 & ANALYTICAL LABORATORY & & & & & & & & 519.500 & $\$ 142,400$ & & & $\$ 161509$ \\
\hline 1400 & RECORD KEEPING & & & & & & & & 57.804 & 528.430 & & & 536.254 \\
\hline
\end{tabular}




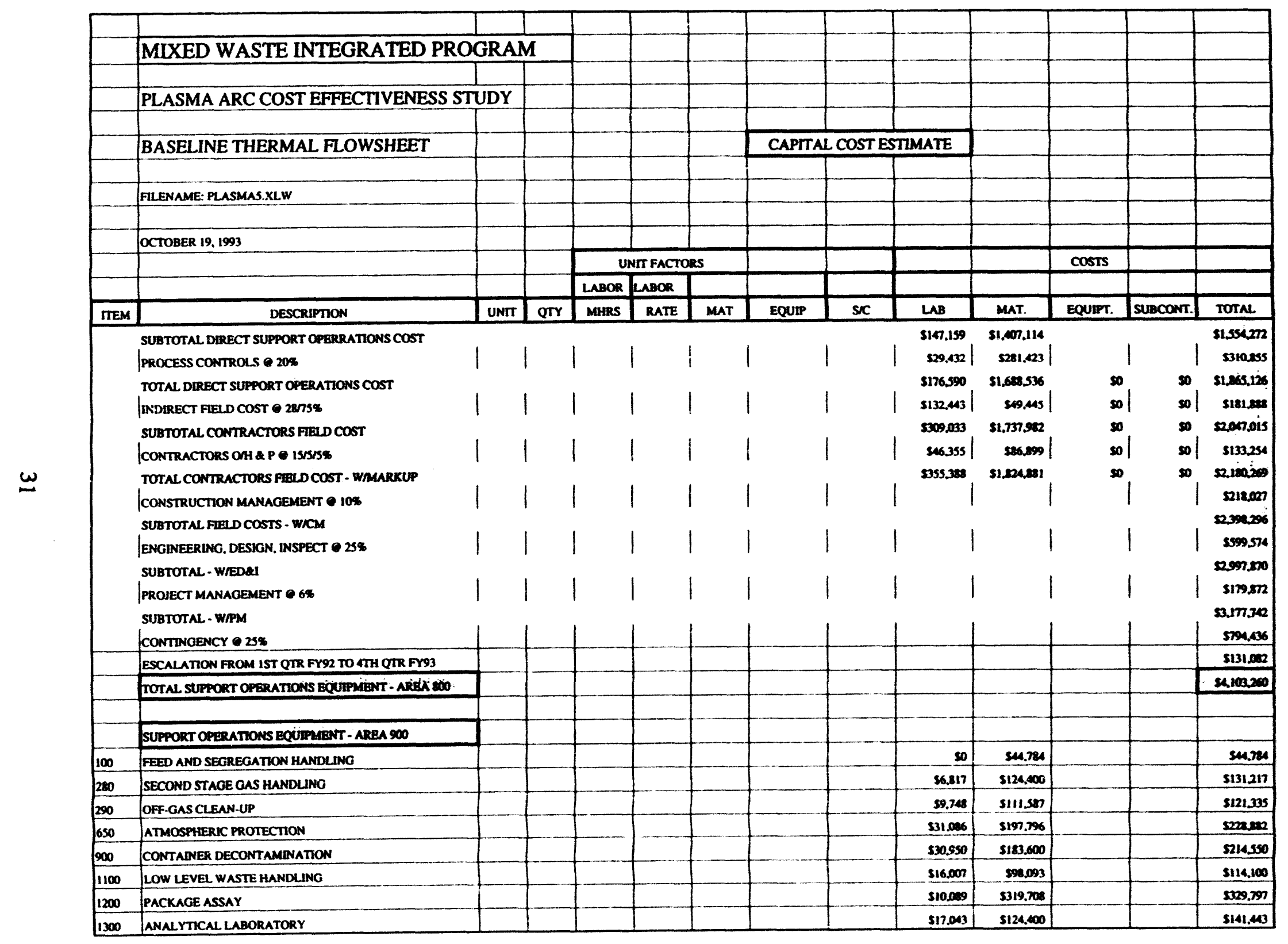




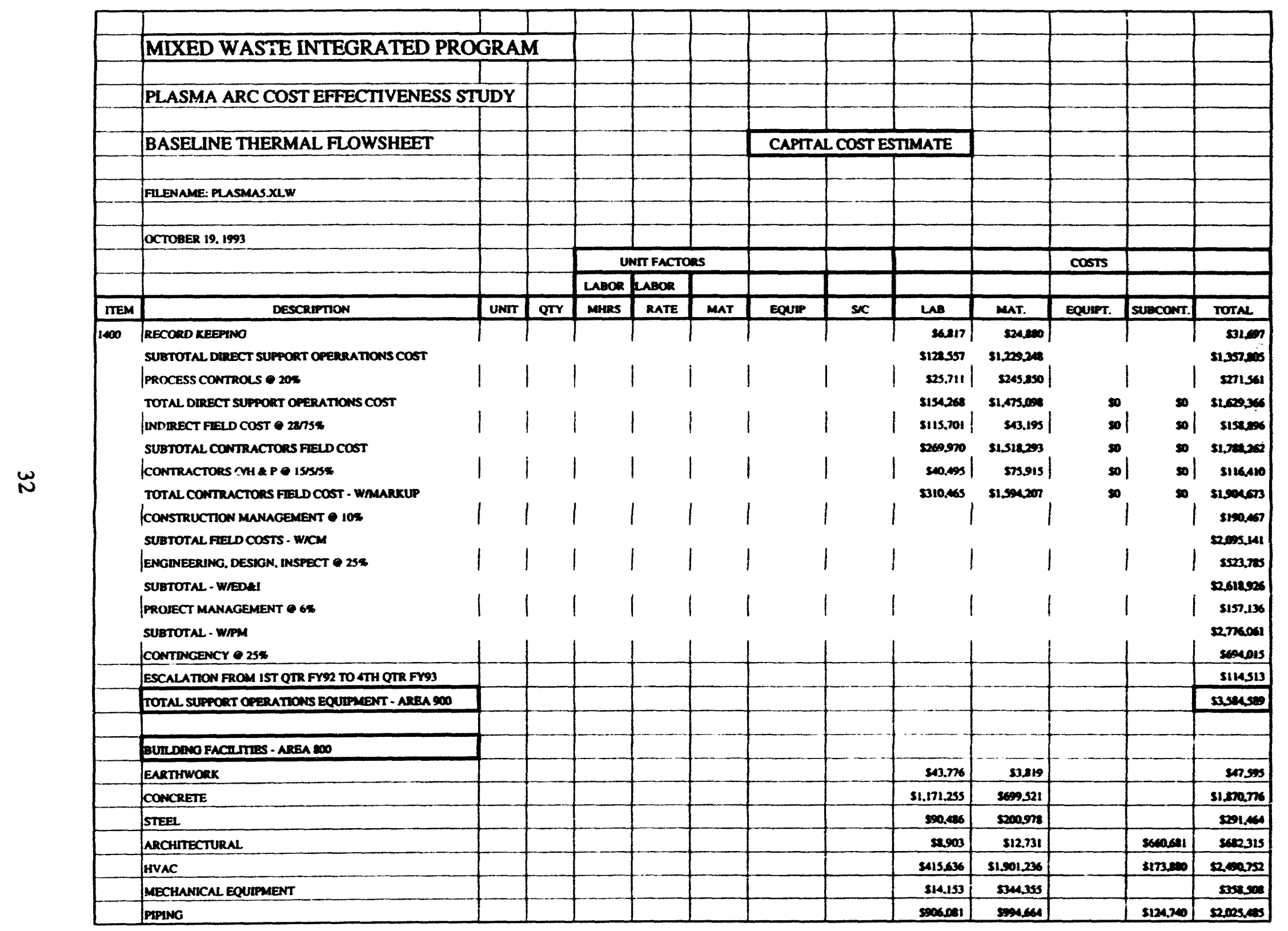




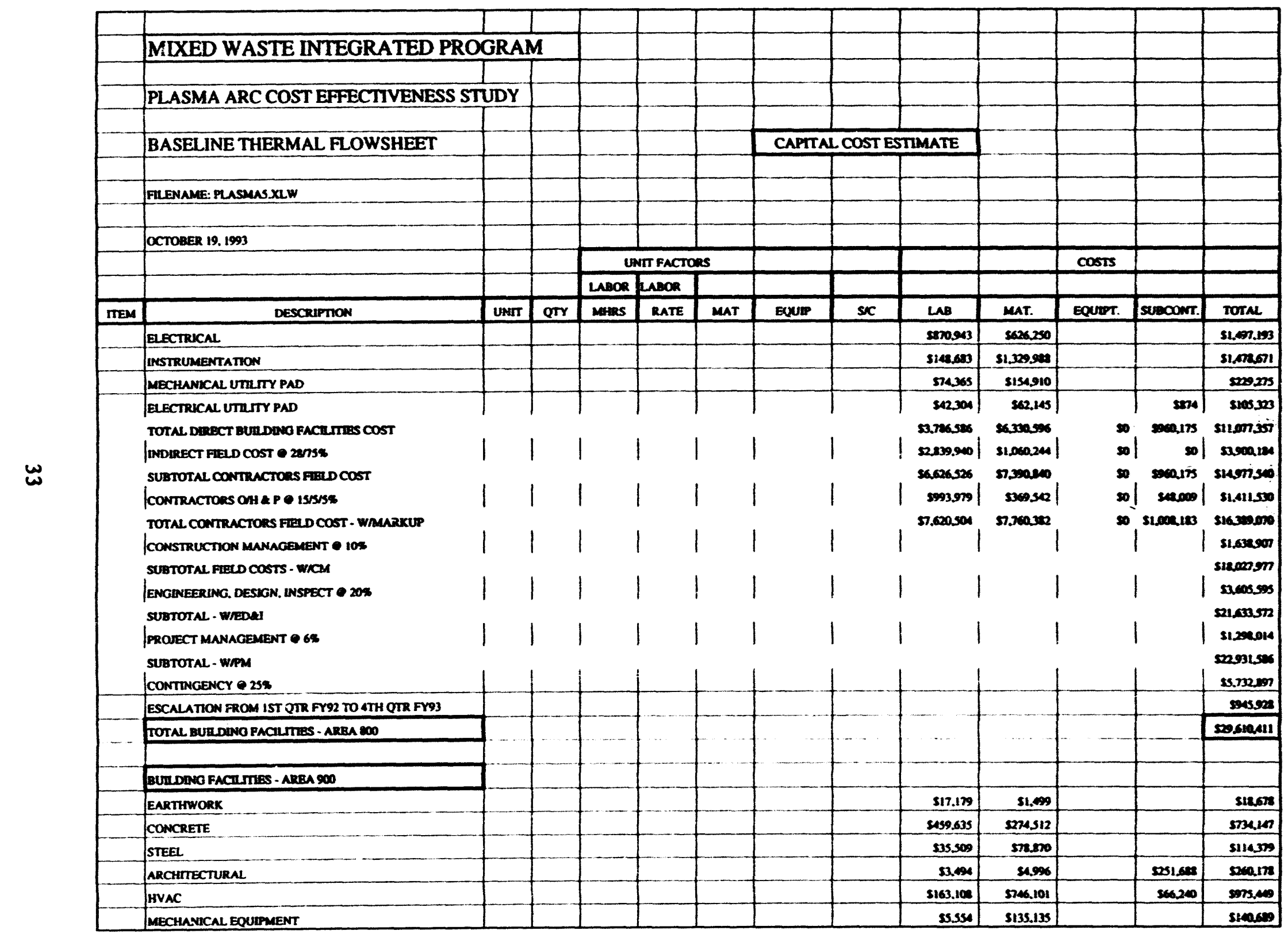




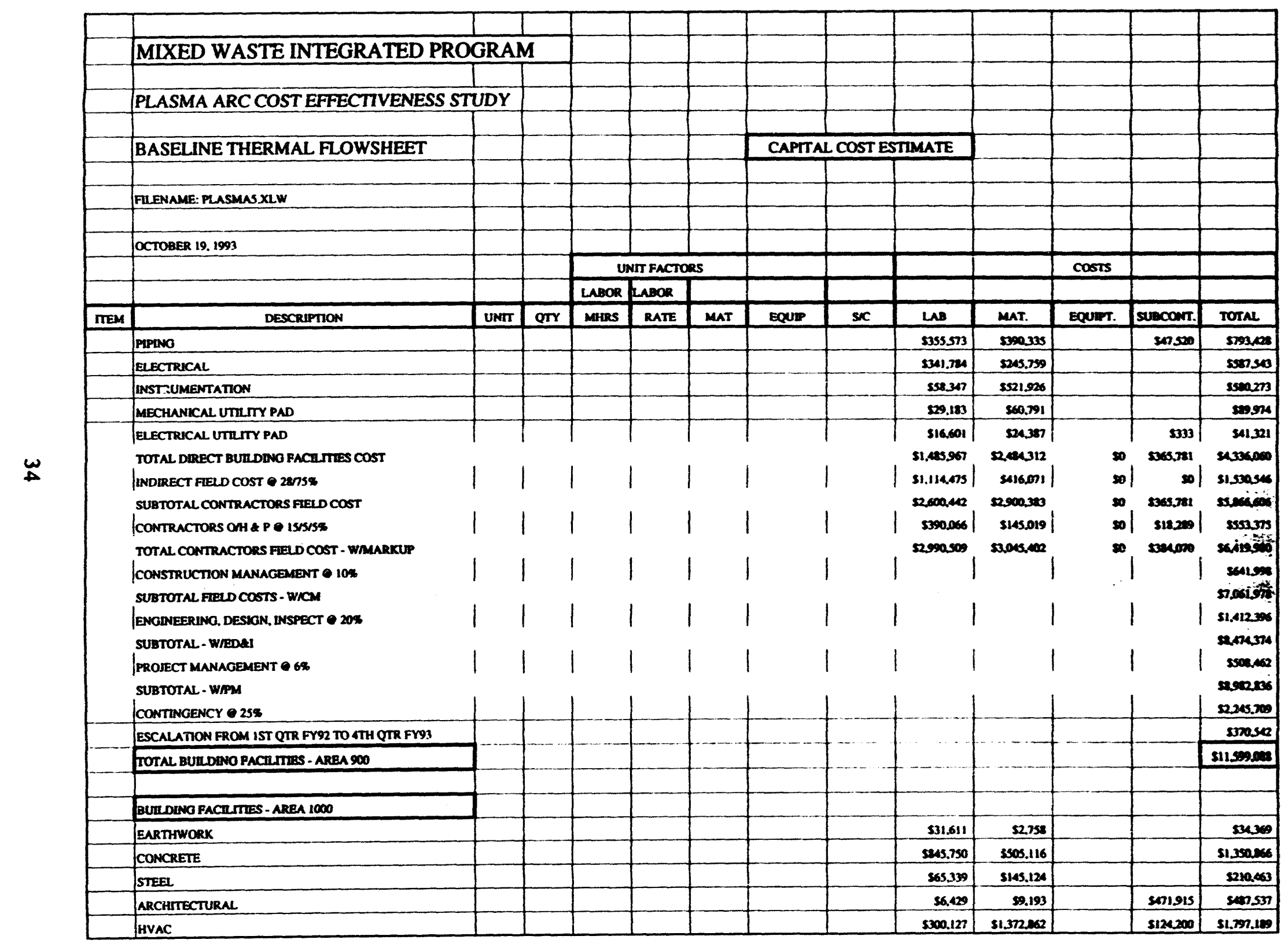




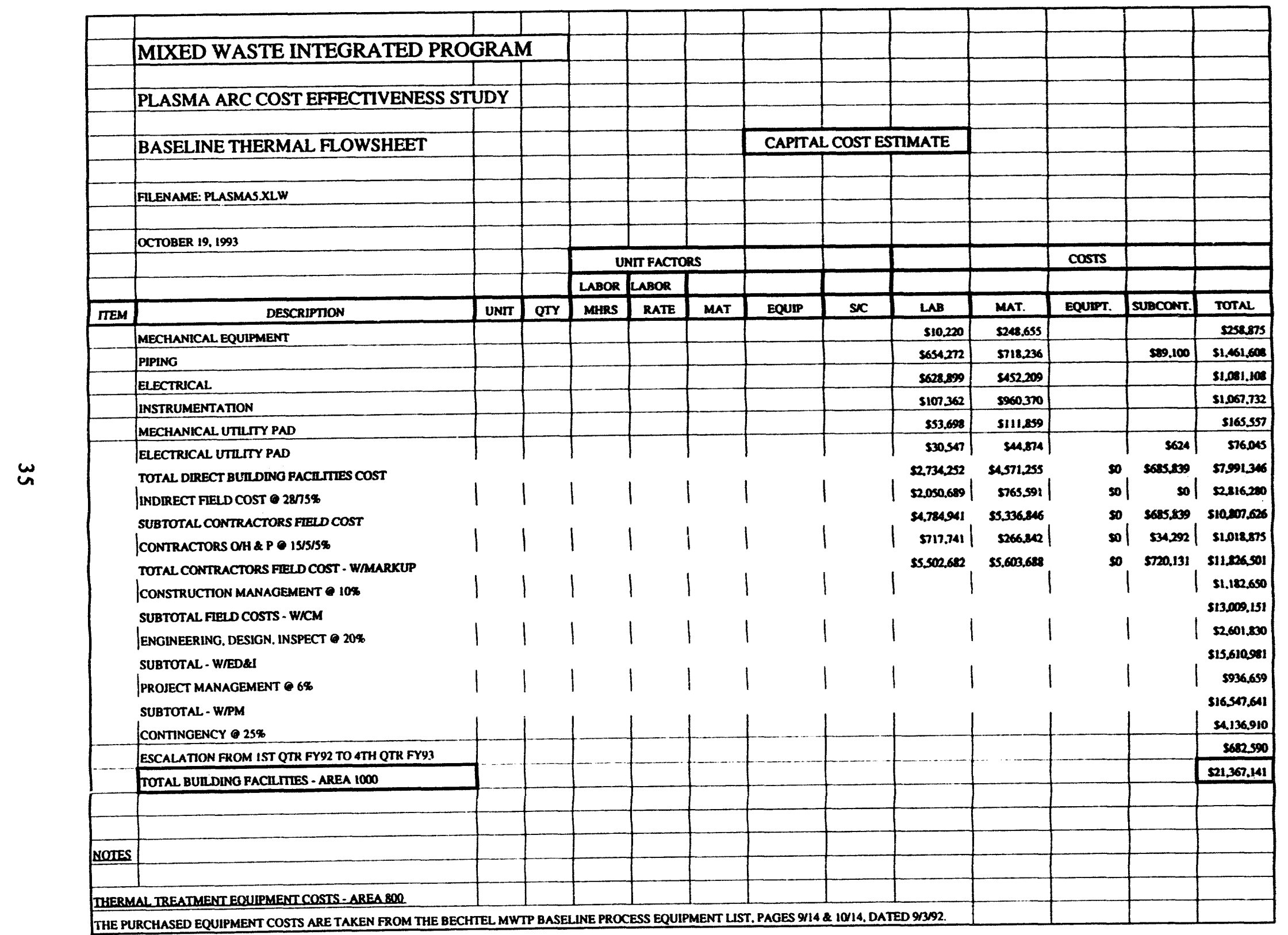




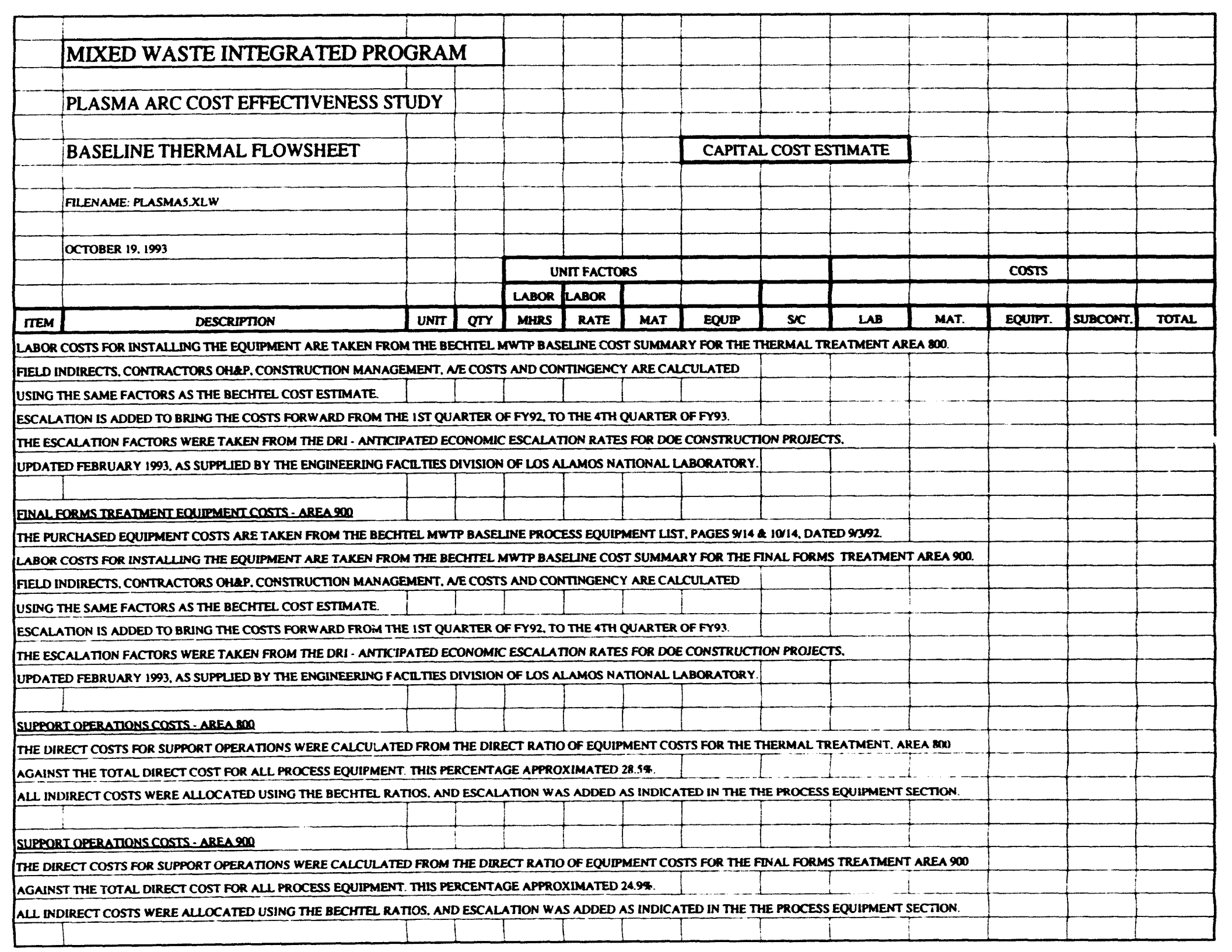




\begin{tabular}{|c|c|c|c|c|c|c|c|c|c|c|c|c|c|}
\hline & \multicolumn{3}{|c|}{ MIXED WASTE INTEGRATED PROGRAM } & & & & & & & & & & \\
\hline & & & & & & & & & & & & & \\
\hline & \multicolumn{2}{|l|}{ PLASMA ARC COST EFFECTIVENESS STUDY } & & & & & & & & & & & \\
\hline & & & & & & & & & & & & & \\
\hline & BASELINE THERMAL FLOWSHEET & & & & & & \multicolumn{3}{|c|}{ CAPITAL COST ESTIMATE } & & & & \\
\hline & & & & & & & & & & & & & \\
\hline & FLLENAME: PLASMAS.XLW & & & & & & & & & & & & \\
\hline & & & & & & & & & & & & & \\
\hline & OCTOBER 19.1993 & & & & & & & & & & & & \\
\hline & & & & \multicolumn{3}{|c|}{ UNT FACTORS } & & & & & costs & & \\
\hline & & & & LABOR I & LABOR & & & & & & & & \\
\hline ITEM & DESCRIPTION & UNIT & QTY & MHRS & RATE & MAT & EQUIP & scc & LAB & MAT. & EQUIPT. & SUBCONT. & TOTAL \\
\hline \multicolumn{14}{|c|}{ BUILDING EACIUITES COSTS- AREA SRO } \\
\hline \multicolumn{14}{|c|}{ USING THE DATA SHOWN IN THE DIRECT COSTS SECTION OF THE BECHTEL MWTP BASEUINE ESTIMATE FOR THE PROCESS BUILDINGS. THE COSTS WERE FACTORED USING } \\
\hline \multirow{2}{*}{\multicolumn{14}{|c|}{$\begin{array}{l}\text { THE RATOO OF BUILDING SPACE ALLOCATION FROM TABLE II OF THE BECHTEL STUDY. THIS FACTOR APPROXIMATED 20.9\% OF THE BULDING FLOOR AREA (13.500 SFFAG.700 } \\
\text { ALL INDIRECT COSTS WERE FACTORED USING THE BECHTEL STUDY FACTORS. }\end{array}$}} \\
\hline \multirow{2}{*}{\multicolumn{14}{|c|}{$\begin{array}{l}\text { ALL INDIRECT COSTS WERE FACTORED USING THE BECHTLL STUDY FACTORS. } \\
\text { ESCALATON WAS ADDED IN THE SAME RATTO AS SHOWN IN THE EQUIPMENT COSTS. }\end{array}$}} \\
\hline \multirow{2}{*}{\multicolumn{14}{|c|}{ ESCALATON WAS ADDED IN THE SAME RATIO AS SHOWN IN THE EQUIPMENT COSTS. }} \\
\hline & & & & & & & & & & & & & \\
\hline \multirow{2}{*}{\multicolumn{14}{|c|}{ 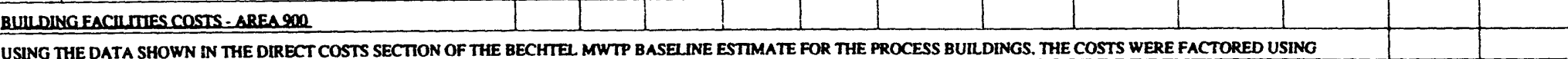 }} \\
\hline USING & & & & & & & & & & & & & \\
\hline \multirow{2}{*}{\multicolumn{14}{|c|}{$\begin{array}{l}\text { THE RATIO OF BULLDING SPACE ALLOCATION FROM TABLE II OF THE BECHTEL STUDY. THIS FACTOR APPROXIMATED 8.25. OF THE BUILDING FLOOR AREA (5.300SF/64,700SF). } \\
\text { ALL INDIRECT COSTS WERE FACTORED USING THE BECHTEL STUDY FACTORS. }\end{array}$}} \\
\hline & & & & & & & & & & & & & \\
\hline \multicolumn{14}{|c|}{ ESCALATION WAS ADDED IN THE SAME RATIO AS SHOWN IN THE EQUIPMENT COSTS. } \\
\hline & & & & & & & & & & & & & \\
\hline \multicolumn{14}{|c|}{ BULDINGEACHIMES COSTS-ABEAUOOO } \\
\hline \multicolumn{14}{|c|}{ USING THE DATA SHOWN IN THE DIRECT COSTS SECTION OF THE BECHTEL MWTP BASELINE ESTIMATE FOR THE PROCESS BUILDINGS. THE COSTS WERE FACTORED USING } \\
\hline \multirow{2}{*}{\multicolumn{14}{|c|}{$\begin{array}{l}\text { THE RATIO OF BUILDING SPACE ALLOCATION FROM TABLE II OF THE BECHTEL STUDY. THIS FACTOR APPROXIMATED IS. } 19 \text {. OF THE BUILD } \\
\text { AUL INDIRECT COSTS WERE FACTORED USING THE BECHTEL STUDY FACTORS. }\end{array}$}} \\
\hline \multirow{2}{*}{\multicolumn{12}{|c|}{ AUL NDIRECT COSTS WERE FACTORED USING THE BECHTEL STUDY FACTORS. }} & & \\
\hline \multicolumn{13}{|c|}{ ESCALATION WAS ADDED IN THE SAME RATTO AS SHOWN IN THE EQUIPMENT COSTS. } & \\
\hline & & & & & & & & & & & & & \\
\hline
\end{tabular}




\section{ALTERNATIVE THERMAL TREATMENT \\ COST ESTIMATE SUMMARY}




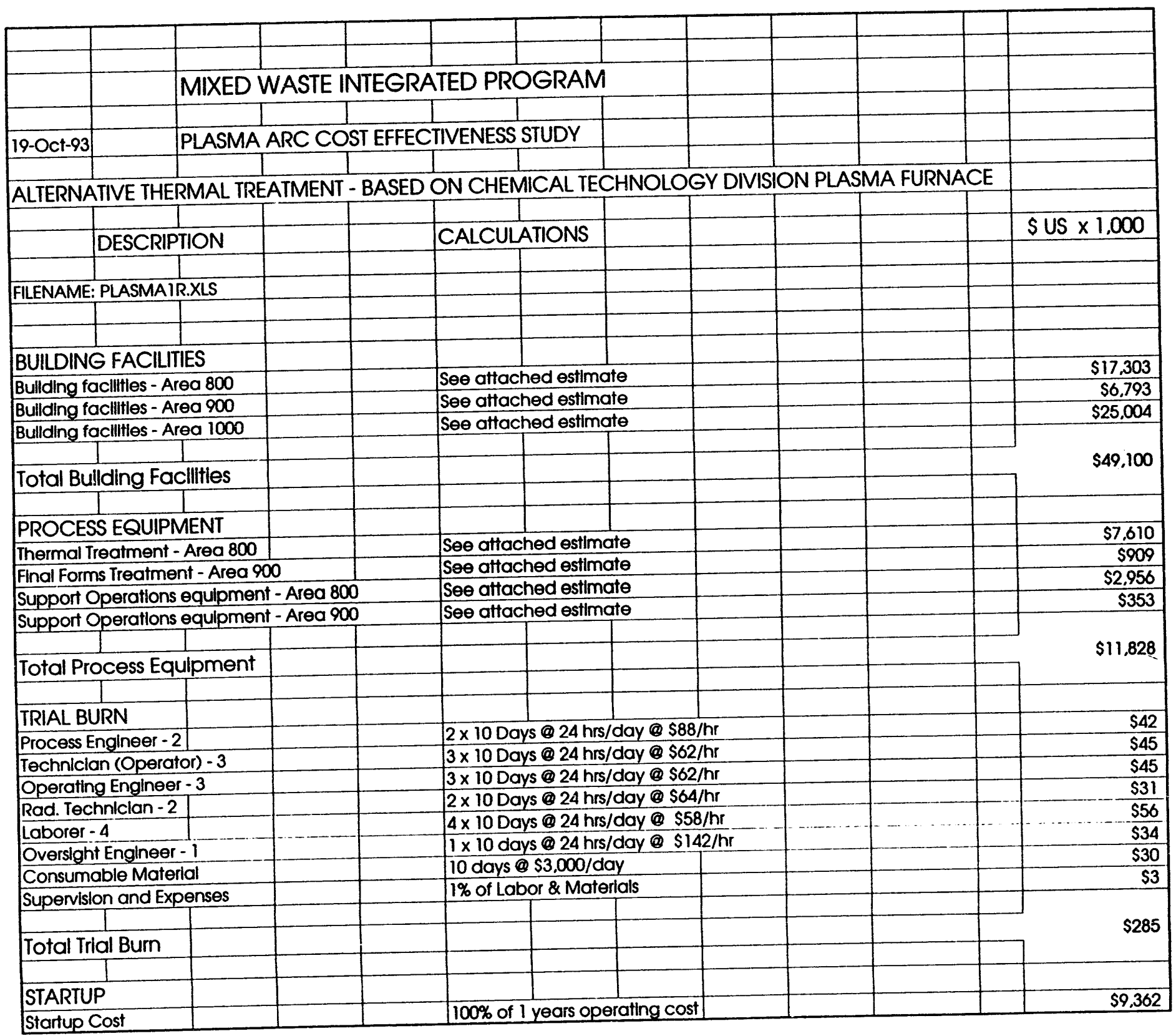




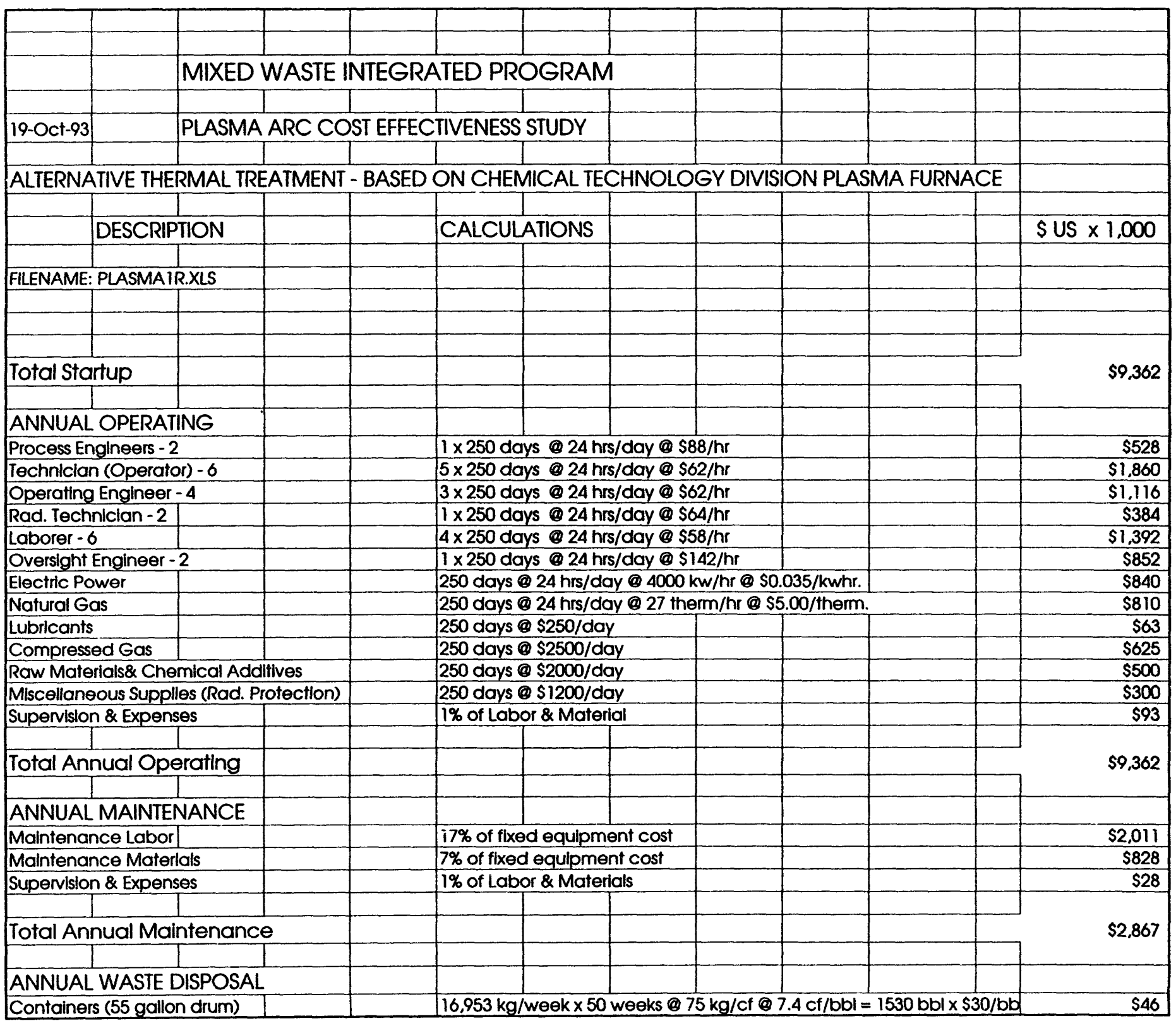


It

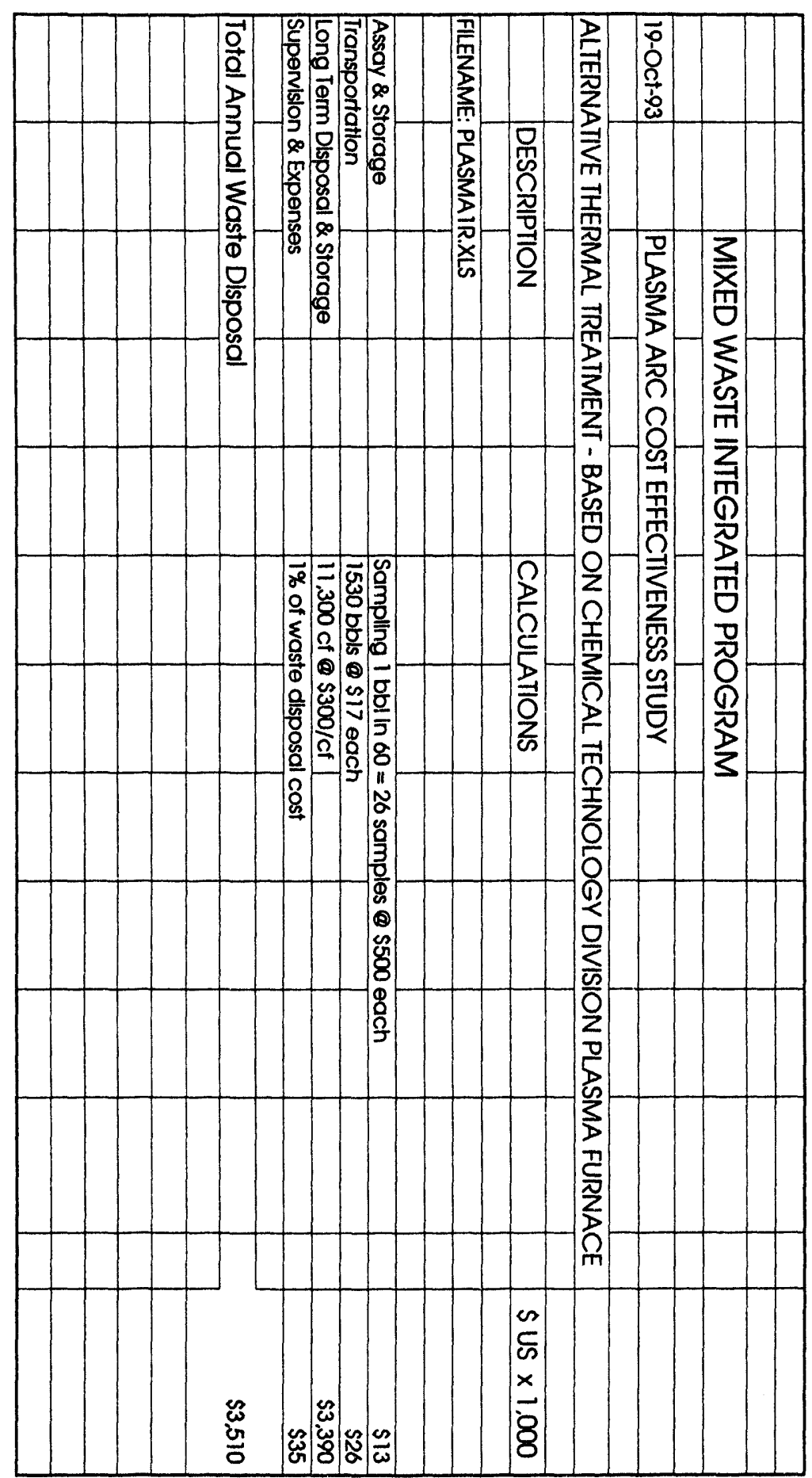


ALTERNATIVE THERMAL TREATMENT

CAPITAL COST ESTIMATE 


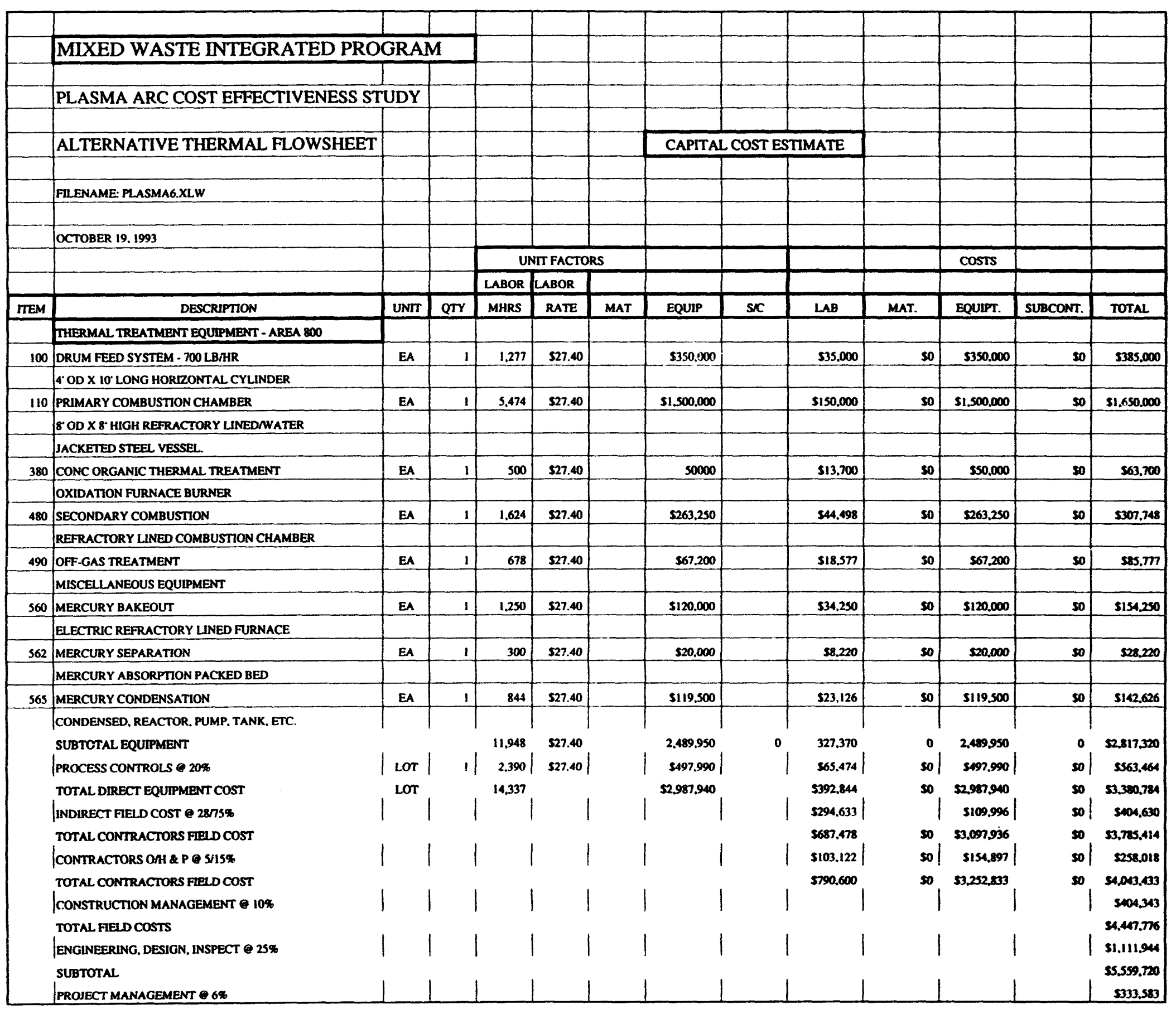




\begin{tabular}{|c|c|c|c|c|c|c|c|c|c|c|c|c|c|}
\hline & MIXED WASTE INTEGRATED PROC & GRAI & & & & & & & & & & & \\
\hline & & & & & & & & & & & & & \\
\hline & PLASMA ARC COST EFFECTIVENESS STU & UDY & & & & & & & & & & & \\
\hline & & & & & & & & & & & & & \\
\hline & ALTERNATIVE THERMAL FLOWSHEET & & & & & & CAPITAL & COST EST & TIMATE & & & & \\
\hline & & & & & & & & & & & & & \\
\hline & FLEENAME: PLASMA6.XLW & & & & & & & & & & & & \\
\hline & & & & & & & & & & & & & \\
\hline & OCTOBER 19. 1993 & & & & & & & & & & & & \\
\hline & & & & & ITFACTOR & & & & & & costs & & \\
\hline & & & & LABOR & LABOR & & & & & & & & \\
\hline mEM & DESCRIPTION & UNIT & QTY & MHRS & RATE & MAT & EQUP & sic & LAB & MAT. & EQuiPT. & susconr. & TOTAL \\
\hline & $\begin{array}{l}\text { SUBTOTAL } \\
\text { CONTINGENCY } 2 \text { 25\% }\end{array}$ & & & & & & & & & & & & $\begin{array}{l}55,893,303 \\
\$ 1,473,326 \\
\end{array}$ \\
\hline & ESCALATION FROM 1ST QTR FY92 TO 4 QTR FY93 & & & & & & & & & & & & $\$ 243.099$ \\
\hline & 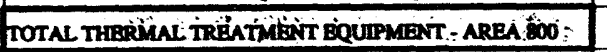 & & & & & & & & & & & & $57,600,25$ \\
\hline & & & & & & & & & & & & & \\
\hline & FINAL FORMS TREATMENT EQOUUPMIENT - ARBA 900 & & & & & & & & & & & & \\
\hline 195 & LEAD POL YMER ENCAPSULATION & EA & 1 & 452 & 527.4 & & $\$ 308.800$ & & $\$ 12.385$ & so & $\$ 3008.200$ & so & 5321.185 \\
\hline 395 & MERCURY POLYMER SOLIDIFICATION & EA & 1 & 36 & \$27.4 & & $\$ 15.700$ & & 5986 & so & $\$ 15.700$ & $\$ 0$ & $\$ 16.686$ \\
\hline 585 & MERCUTY DISTLLLATION & EA & 11 & 92 & 527.4 & & $\$ 24.000$ & & 52.521 & sol & 524.000 & sol & 526.521 \\
\hline & $\begin{array}{l}\text { SUBTOTAL DRECT EQUTPMENT COST } \\
\text { PROCESS CONTROLS Q 20\% }\end{array}$ & LOT & 11 & $\begin{array}{l}580 \\
116\end{array}$ & $\begin{array}{l}\$ 27.40 \\
\$ 27.70\end{array}$ & so & $\begin{array}{l}\$ 348.500 \\
\$ 69.700\end{array}$ & so & $\begin{array}{c}\$ 15.892 \\
\$ 3.213\end{array}$ & sol & $\begin{array}{c}5348,500 \\
569.700\end{array}$ & sol & $\begin{array}{r}5364,392 \\
572.913\end{array}$ \\
\hline & $\begin{array}{l}\text { TOTAL DIRECT EQUIPMENT COST } \\
\mid \text { INDIRECT FELL COST } 2 \text { 2875\% }\end{array}$ & LOT & & 696 & & & $\$ 418,200$ & & $\begin{array}{l}\mathbf{s 1 9 . 1 0 5} \\
\mathbf{s 1 4 , 3 2 9}\end{array}$ & so & $\begin{array}{r}\$ 18.200 \\
\$ 5.349\end{array}$ & sol & $\begin{array}{r}5137.305 \\
519.673\end{array}$ \\
\hline & SUBTOTAL CONTRACTORS FEELD COST & & & 1 & 1 & & & & $\mathbf{5 3 3 . 4 3 4}$ & so & 5023.549 & 50 & s456,9en \\
\hline & CONTRACTORS O/H \& PQ S/IS\% & & 1 & 1 & 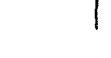 & 1 & & & 55.015 & sol & 521.17 & sol & 526.193 \\
\hline & $\begin{array}{l}\text { TOTAL CONTRACTORS FEID COST - WMARKUP } \\
\text { CONSTRUCTION MANAGEMENT } 10 \%\end{array}$ & & 1 & 1 & | & 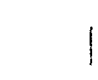 & & & \$ & 1 & $\operatorname{sen} .727$ & 50 & $\begin{array}{r}5483.176 \\
548.318\end{array}$ \\
\hline & SUBTUTAL FELD COSTS - W/CM & & & & & & & & & & & & $\$ 531.494$ \\
\hline & |ENGINEERING, DESIGN, INSPECT $25 \%$ & & & 1 & | & & 1 & & & 1 & & 1 & $\$ 132.873$ \\
\hline & SUBTOTAL - W/EDAI & & & & & & & & & 1 & & & $\$ 664.367$ \\
\hline & PROJECT MANAGEMENT $6 \%$ & & 1 & 1 & & & & & 1 & 1 & & 1 & 539.862 \\
\hline & $\begin{array}{l}\text { SUBTOTAL - W/PM } \\
\text { CONTINGENCY } 25 \%\end{array}$ & & & & & & & & & & & & $\begin{array}{l}5704.209 \\
5176.057\end{array}$ \\
\hline & ESCALATION FROM IST QTR FY92 TO 4 QTR FY93 & & & & & & & & & & & & $\$ 29.009$ \\
\hline & TOTAL FINAL FORMS TREATMENT BQUIPMENT - AREA 900 & & & & & & & & & & & & 5009336 \\
\hline & & & & & & & & & & & & & \\
\hline & SUPPORT OPERATIONS EQUIPMENT - AREA 800 & & & & & & & & & & & & \\
\hline 100 & FEED AND SEGREGATION HANDLING & & & & & & & & 50 & 536.936 & & & 536936 \\
\hline 280 & SECOND STAGE GAS HANDLING & & & & & & & & $\$ 5.622$ & $\$ 102.000$ & & & $51002 m$ \\
\hline 290 & OFF-GAS CLEAN-UP & & & & & & & & $\$ 8.040$ & 592,032 & & & sioe.0n \\
\hline
\end{tabular}




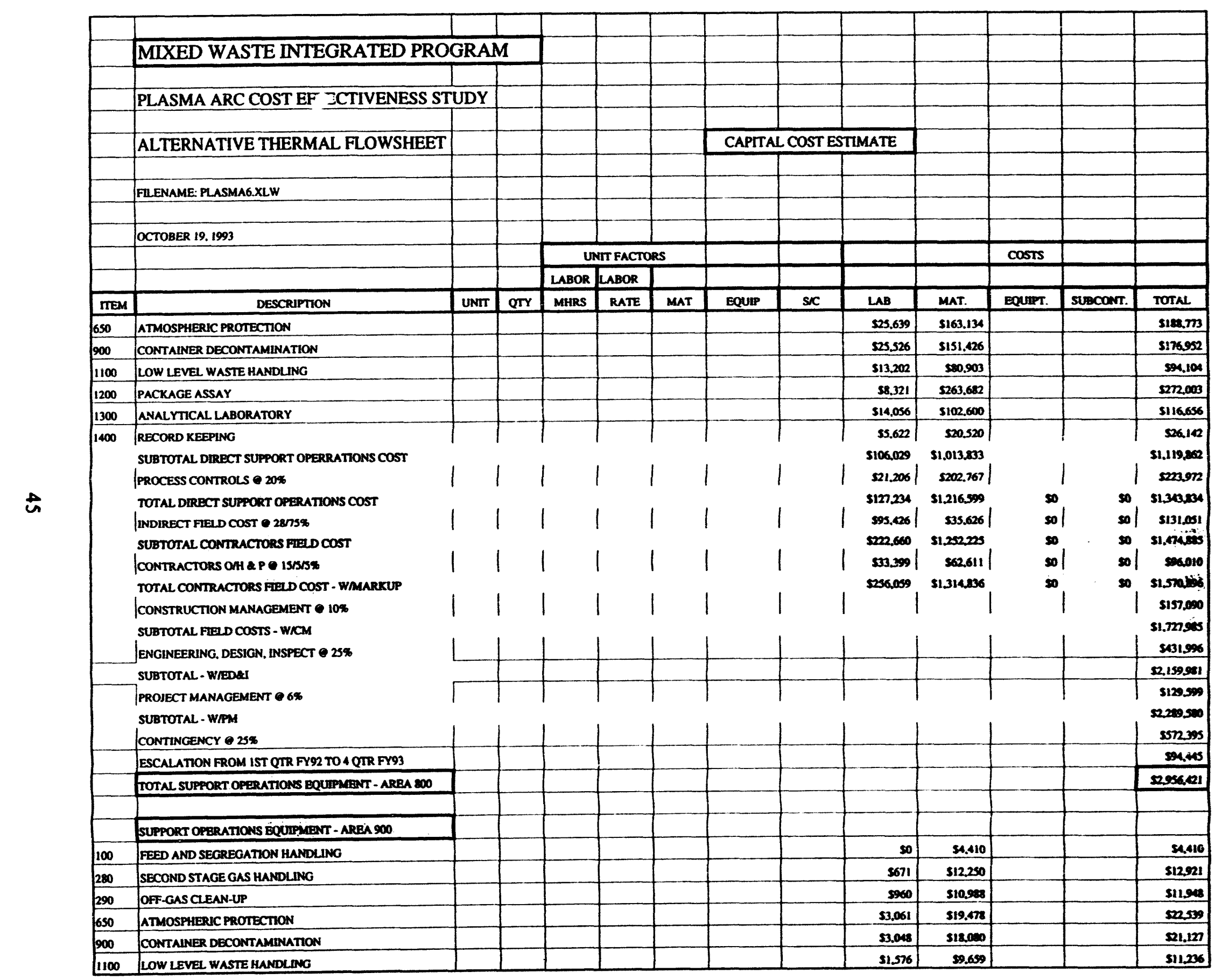




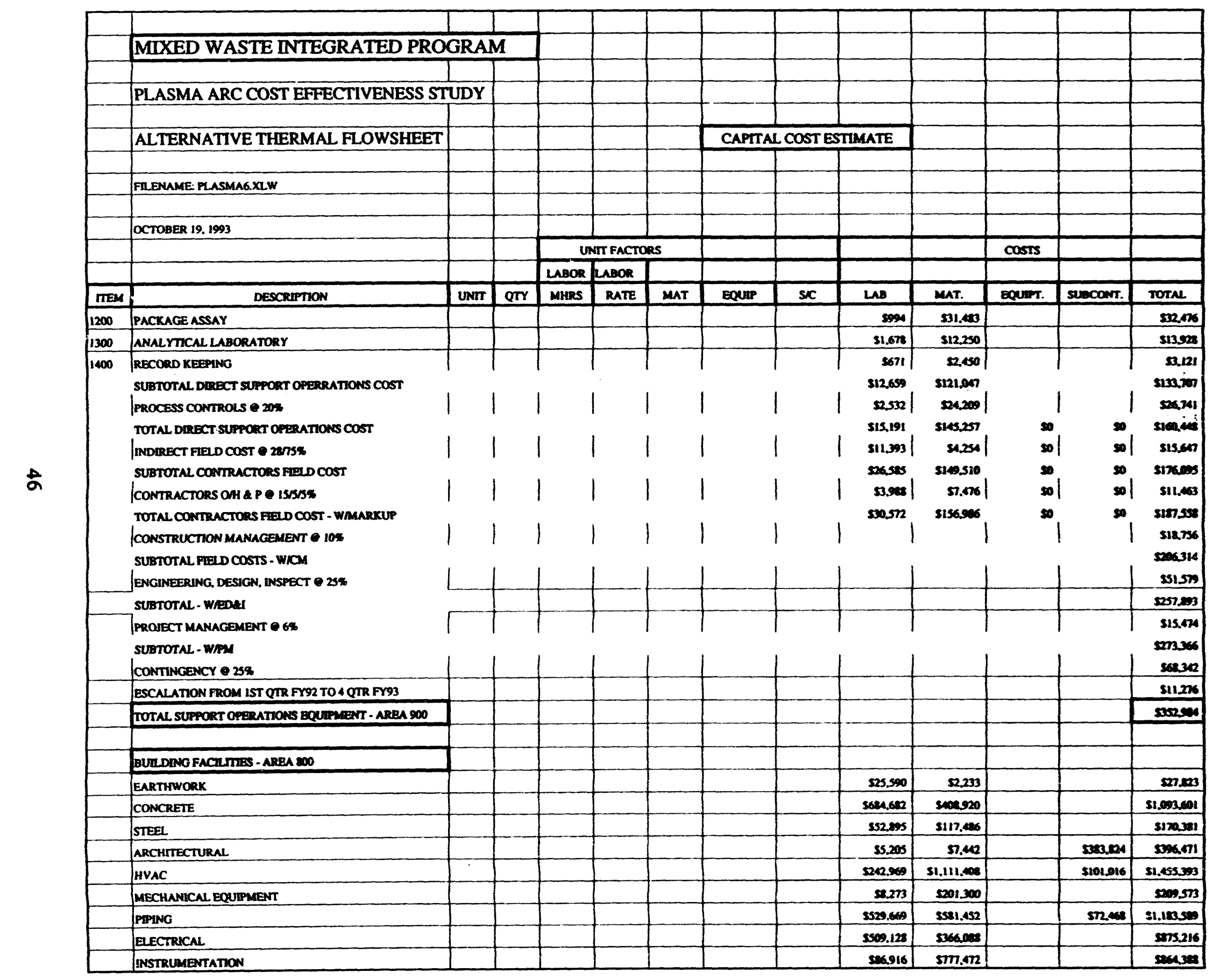




\begin{tabular}{|c|c|c|c|c|c|c|c|c|c|c|c|c|c|}
\hline & & & & & & & & & & & & & \\
\hline & \multicolumn{3}{|c|}{ MIXED WASTE INTEGRATED PROGRAM } & & & & & & & & & & \\
\hline & \multirow{2}{*}{\multicolumn{2}{|c|}{ PLASMA ARC COST EFFECTIVENESS STUDY }} & & & & & & & & & & & \\
\hline & & & & & & & & & & & & & \\
\hline & & & & & & & & & & & & & \\
\hline & & & & & & & \multicolumn{3}{|c|}{ CAPITAL COST ESTIMATE } & & & & \\
\hline & FLENAME: PLASMAG XLW & & & & & & & & & & & & \\
\hline & & & & & & & & & & & & & \\
\hline & OCTOBER 19. 1993 & & & & & & & & & & & & \\
\hline & & & & & & & & & & & & & \\
\hline & & & & & IrFACTO & & & & & & costs & & \\
\hline mEM & DESCRIPTION & & & LABOR & LABOR & & & & & & & & \\
\hline & \begin{tabular}{|l} 
DESCRIPTION \\
MECHANACAL UTILITY PAD \\
\end{tabular} & UNII & QTY & MHes & RATE & MAT & EOUP & sx & LAB & Mat. & EQunt. & sueconr. & Total \\
\hline & $\begin{array}{l}\text { MECHANACAL UTILTTY PAD } \\
\text { ELECTRICAL UTILTYY PAD }\end{array}$ & & & & & & & & 43471.528 & sosss.n. & & & s134.027 \\
\hline & $\begin{array}{l}\text { TELECTRICAL UTLITY PAD } \\
\text { TOTAL DIRECT BUILDNG FACTI MTES COST }\end{array}$ & & & & & & & & 524,730 & $\$ 36.328$ & & $\operatorname{sses} \mid$ & 261.565 \\
\hline & $\begin{array}{l}\text { TOTAL DIRECT BULLDING FACHITIES COST } \\
\text { |INDIRECT FIELD COST } 2 \text { 28TS\% }\end{array}$ & & & | & & & & & $\begin{array}{l}\$ 2.213 .529 \\
\$ 1.660 .147\end{array}$ & $\begin{array}{l}53.700 .034 \\
2619.708\end{array}$ & $\infty 01$ & $\begin{aligned} 5057.816 \\
50 \mid\end{aligned}$ & $\begin{array}{l}24072009 \\
22279.935\end{array}$ \\
\hline & SUBTOTAL CONTRACTORS FIED COST & & & & & & & & 23.87.67s & $\sin 320,42$ & $\infty$ & 5557816 & sursises \\
\hline & |CONTRACTORS OM \& P ISISAS & & & 1 & & & & & $\$ 581.051$ & $\$ 216.024$ & sol & mas $\mid$ & $\operatorname{sensess}$ \\
\hline & TOTAL CONTRACTORS FELD COST - WMARKUP & & & & & & & & S4,454,727, & $34,536,466$ & $s 0$ & $508 . \pi$ & sisios \\
\hline & $\begin{array}{l}\text { |CONSTRUCTION MANAGEMENT } \text { IO\% } \\
\text { SUBTOTAL FIEL COSTS - WICM }\end{array}$ & & & & & & & & & & & & $\operatorname{sinsting}$ \\
\hline & JENGENEERING, DESIGN, INSPECT $20 \%$ & & & I & & & & & 1 & 1 & & & 2210029 \\
\hline & SUBTOTAL - W/EDSI & & & 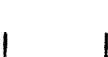 & & & & & & 1 & & & s12cat:sin \\
\hline & |Project management e $6 \%$ & & & 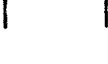 & & & & & 1 & 1 & & 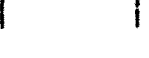 & 5289,403 \\
\hline & $\begin{array}{l}\text { SUBTOTAL - WIPM } \\
\text { CONTINGENCY E 25\% }\end{array}$ & & & & & & & & & & & & 23.300010 \\
\hline & ESCALATION FROM IST QTR FY92 TO4 QTR FY93 & & & & & & & & & & & & 555272 \\
\hline & TOTAL BUM DINO FACRIMES - AREA 800 & & & & & & & & & & & & $\operatorname{sin,302000}$ \\
\hline & & & & & & & & & & & & & \\
\hline & BURDONG FACRITIES - AREA 900 & & & & & & & & & & & & \\
\hline & EARTHWORK & & & & & & & & $\$ 10.047$ & $\operatorname{sen}$ & & & s10.924 \\
\hline & CONCRETE & & & & & & & & $5268.2 \mathrm{~m}$ & s160.351 & & & $\mathbf{s} 29.373$ \\
\hline & STEE- & & & & & & & & 520.768 & $\mathbf{s e 6 . 1 2 8}$ & & & $360 \mathrm{mas}$ \\
\hline & ARCHITECTURAL & & & & & & & & $\$ 2.003$ & $\$ 2.922$ & & siso.6en & $\$ 155.604$ \\
\hline & HVAC & & & & & & & & 595.395 & 5436.364 & & 839.661 & 2571,421 \\
\hline & MECHANICAL EQUUPMENT & & & & & & & & 53.248 & 579.035 & & & 5220 \\
\hline & PAPING & & & & & & & & 5200.960 & $\operatorname{sen} 291$ & & 520,453 & $\operatorname{sen}, x$ \\
\hline & ELECTRICAL & & & & & & & & 199095.4178 & 143734.438 & & & $\mathbf{5 3 3 , 6 3 0}$ \\
\hline & INSTRUMENTATION & & & & & & & & 534.125 & 5300.254 & & & 5339379 \\
\hline & MECHANICAL UTLITY PAD & & & & & & & & 17067.9196 & 35554.254 & & & $\$ 52.622$ \\
\hline & ELECTRICAL UTUITY PAD & & & & & & & & 99.710 & $\$ 314.263$ & & $\mathbf{s 1 9 9}$ & 526.17 \\
\hline & TOTAL DRRPCT BÜLNNO FACTIIIES COST & & & & & & & & 5069.002 & $\$ 1.452973$ & so & 2819.011 & 5251.00 \\
\hline
\end{tabular}




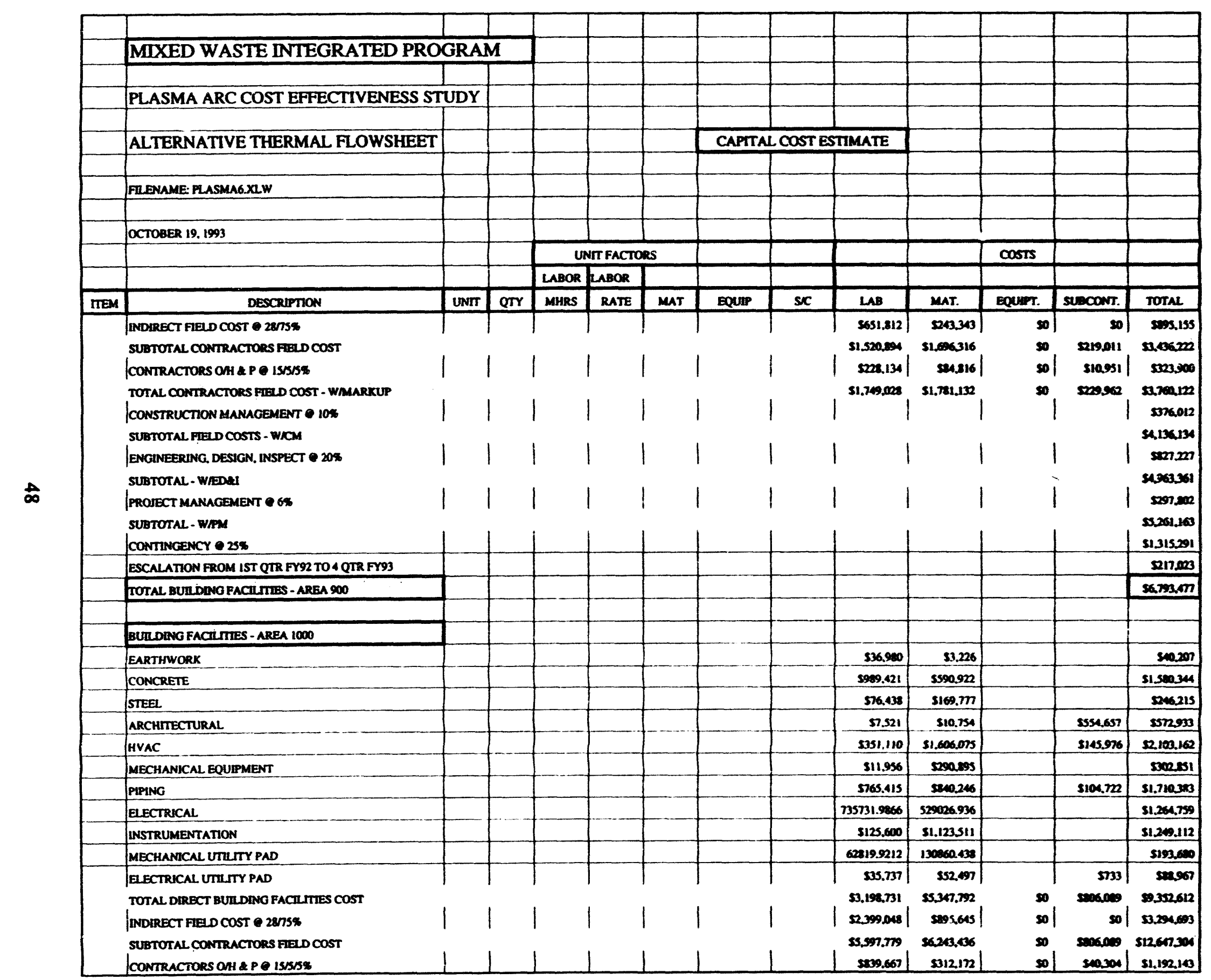




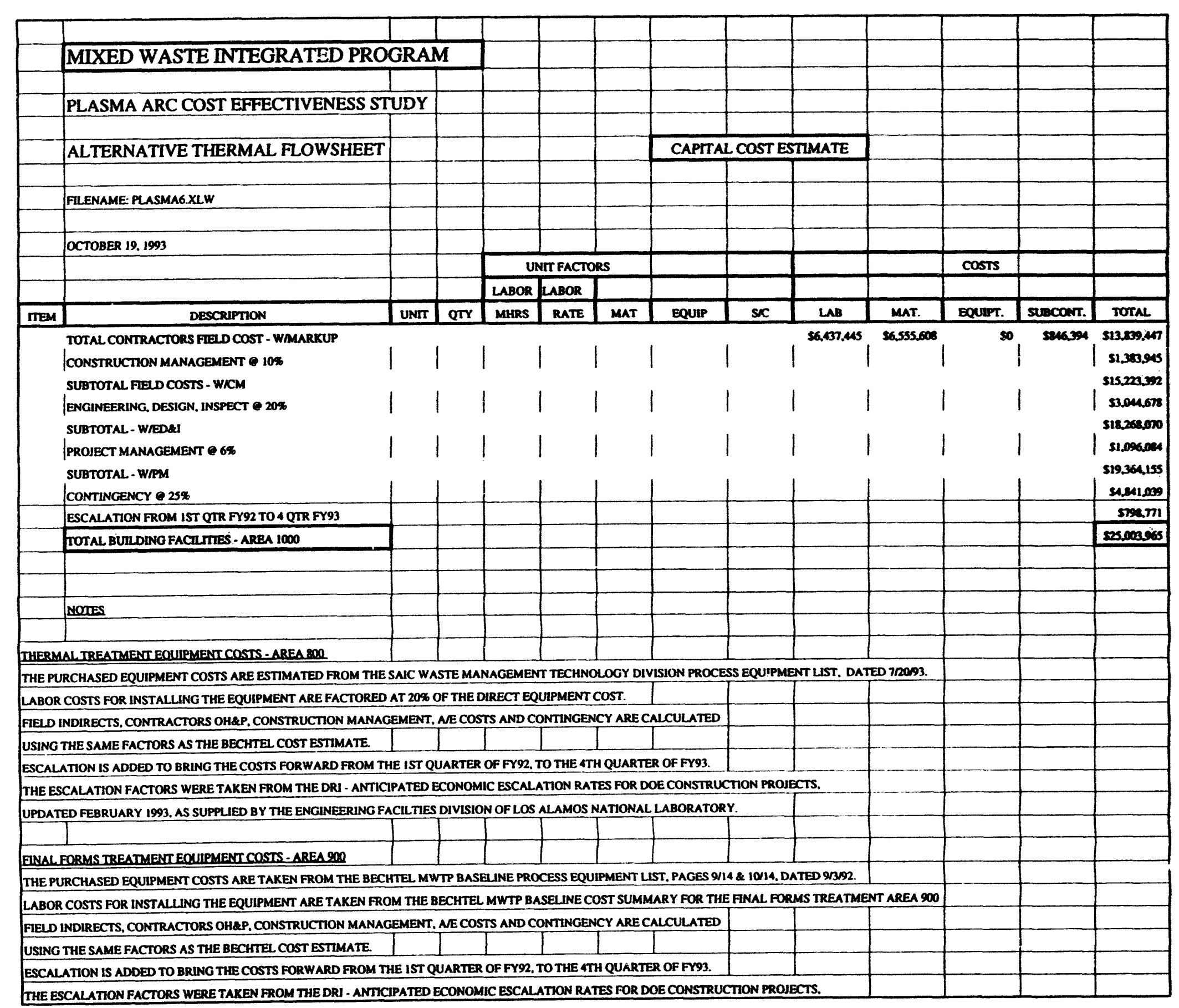




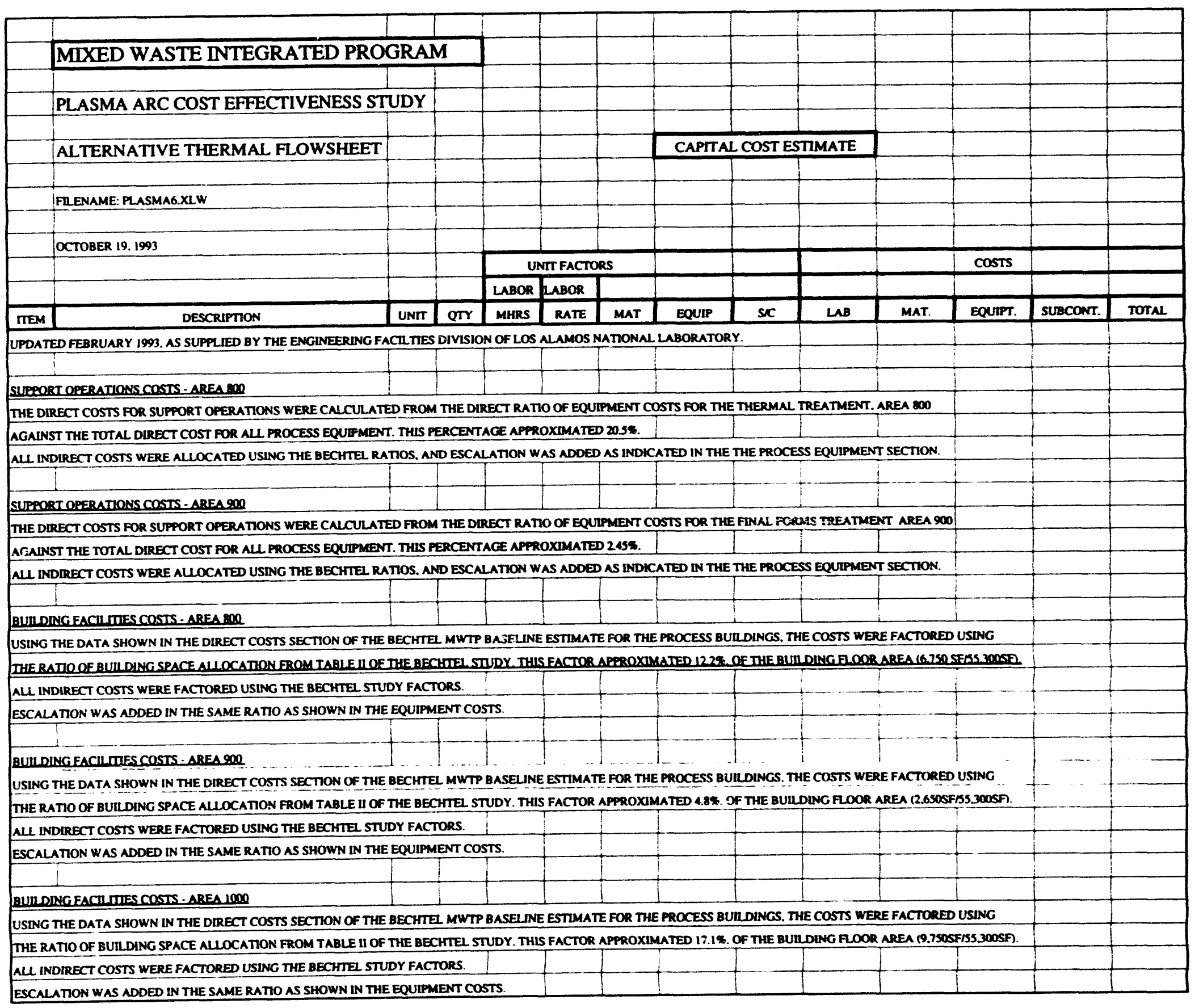



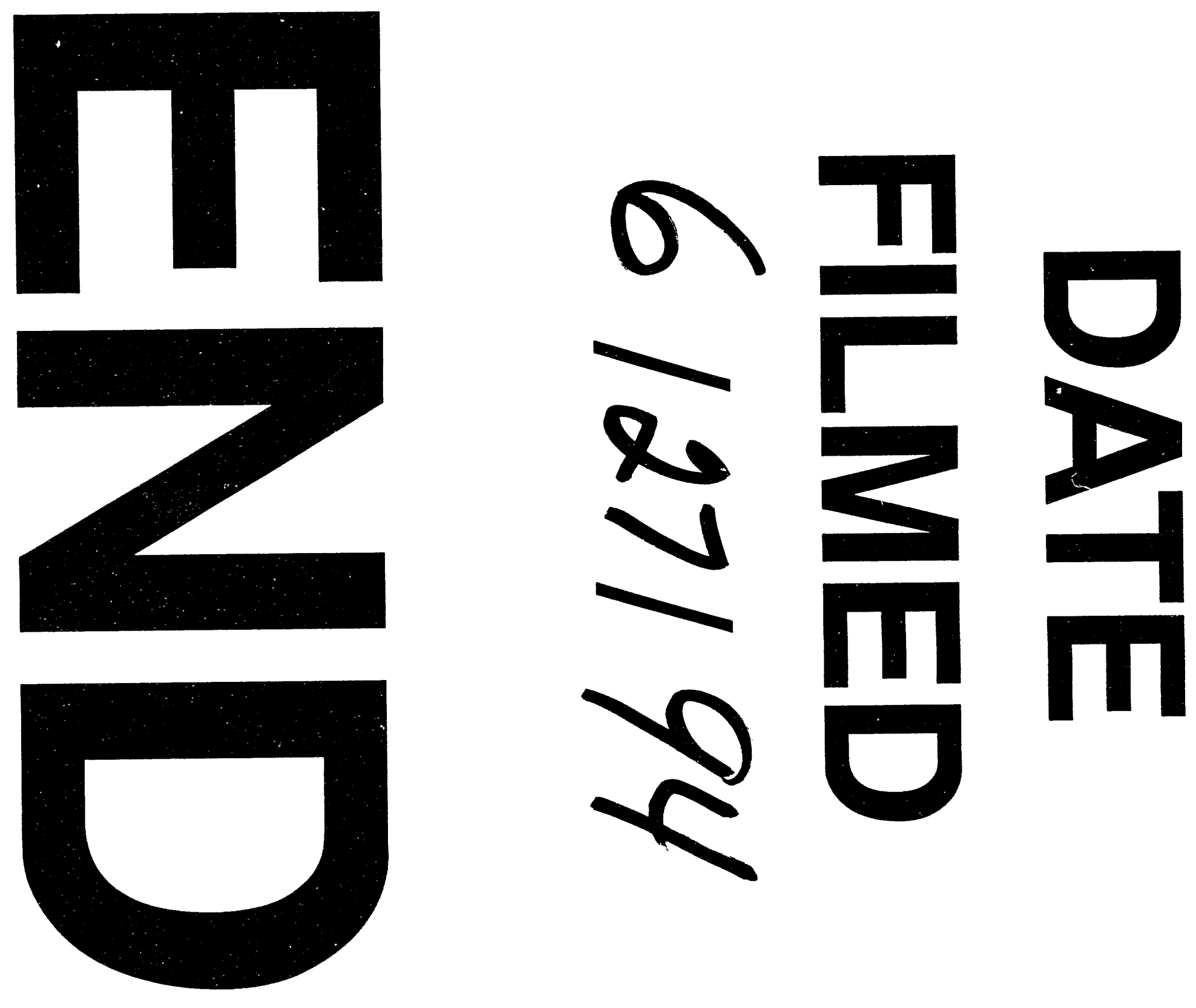
